\title{
Patient-centred outcomes are under- reported in the critical care burns literature: a systematic review
}

\author{
Karthik Venkatesh ${ }^{1,2^{*}}$ (D) Alice Henschke ${ }^{3,4}$, Richard P. Lee ${ }^{1,4}$ and Anthony Delaney ${ }^{1,4,5}$
}

\begin{abstract}
Background: Developments in the care of critically ill patients with severe burns have led to improved hospital survival, but long-term recovery may be impaired. The extent to which patient-centred outcomes are assessed and reported in studies in this population is unclear.

Methods: We conducted a systematic review to assess the outcomes reported in studies involving critically ill burns patients. Randomised controlled trials (RCTs) and cohort studies on the topics of fluid resuscitation, analgesia, haemodynamic monitoring, ventilation strategies, transfusion targets, enteral nutrition and timing of surgery were included. We assessed the outcomes reported and then classified these according to two suggested core outcome sets.

Results: A comprehensive search returned 6154 studies; 98 papers met inclusion criteria. There were 66 RCTs, 19 clinical studies with concurrent controls and 13 interventional studies without concurrent controls. Outcome reporting was inconsistent across studies. Pain, reported using the visual analogue scale, fluid volume administered and mortality were the only outcomes measured in more than three studies. Sixty-six studies (67\%) had surrogate primary outcomes. Follow-up was poor, with median longest follow-up across all studies 5 days (IQR 3-28). When compared to the suggested OMERACT core outcome set, 53\% of papers reported on mortality, $28 \%$ reported on life impact, 30\% reported resource/economic outcomes and 95\% reported on pathophysiological manifestations. Burns-specific Falder outcome reporting was globally poor, with only $4.3 \%$ of outcomes being reported across the 98 papers.

Conclusion: There are deficiencies in the reporting of outcomes in the literature pertaining to the intensive care management of patients with severe burns, both with regard to the consistency of outcomes as well as a lack of focus on patient-centred outcomes. Long-term outcomes are infrequently reported. The development and validation of a core outcome dataset for severe burns would improve the quality of reporting.
\end{abstract}

Keywords: Burn, Thermal injury, Critical care, Core Outcome, Review, Patient-centred

\footnotetext{
* Correspondence: Karthik.venkatesh@health.nsw.gov.au

'Malcolm Fisher Department of Intensive Care, The Royal North Shore

Hospital, St Leonards, NSW 2065, Australia

${ }^{2}$ The University of New South Wales, Kensington, Sydney, NSW, Australia

Full list of author information is available at the end of the article
}

(C) The Author(s). 2022 Open Access This article is licensed under a Creative Commons Attribution 4.0 International License, which permits use, sharing, adaptation, distribution and reproduction in any medium or format, as long as you give appropriate credit to the original author(s) and the source, provide a link to the Creative Commons licence, and indicate if changes were made. The images or other third party material in this article are included in the article's Creative Commons licence, unless indicated otherwise in a credit line to the material. If material is not included in the article's Creative Commons licence and your intended use is not permitted by statutory regulation or exceeds the permitted use, you will need to obtain permission directly from the copyright holder. To view a copy of this licence, visit http://creativecommons.org/licenses/by/4.0/ The Creative Commons Public Domain Dedication waiver (http://creativecommons.org/publicdomain/zero/1.0/) applies to the data made available in this article, unless otherwise stated in a credit line to the data. 


\section{Introduction}

Severe burn injury is potentially catastrophic for a patient, often requiring prolonged intensive care support and causing significant acute and long-term complications [1]. The ultimate goal of burn care is to restore a patient to a functional level as close to pre-injury status as possible. In the acute phase of severe burn injury, intensive care interventions are focussed on resuscitation and largely short-term based goals. The extent to which these initial interventions impact on long term patientcentred outcomes is unclear.

The quality and consistency of outcome reporting in studies of patients with severe burn injury has been questioned, with numerous calls for a core outcome set (COS) to improve reporting [2-4]. Core outcomes are defined as an 'agreed, standardised collection of outcomes measured and reported in all trials for a specific clinical area' [5], which facilitates comparison of findings between clinical trials and improves the body of evidence in a particular field. In 1992, the Outcomes Measures in Rheumatoid Arthritis Clinical Trials (OMERACT) group developed a comprehensive framework to establish a set of core outcomes in clinical trials of rheumatology, which has seen significant improvement in outcome reporting in rheumatological trials [6]. The full framework has been well described by Boers et al. [7]. The framework consists of four key domains, from which outcomes relevant to each must be reported. The domains are mortality, life impact (patient-centred outcomes including quality of life, pain, functional status), economic/resource use and pathophysiological manifestations (such as clinical and biochemical outcomes). Part of the success of the framework has been emphasising patient-centred outcomes into COS development, in order to ensure that outcomes relevant to the patients are given importance [8]. Given the broad applicability of these domains to other medical fields, the framework has been implemented into other specialities including cardiothoracic surgery, maternity care, inflammatory bowel disease and paediatric illnesses [9-11]. The implementation of core outcome sets into critical care research has been lagging, and there have been a number of critical care research projects working on COS development, many of which have been guided by the OMERACT framework and its broadly relevant domains [11].

The consistency of outcome reporting in the literature pertaining to the intensive care management of severe burns is unclear. Furthermore, the extent to which patient-centred outcomes are reported in this literature is unclear. Therefore, we performed a systematic review to assess the nature of outcome reporting in studies of critically ill patients with severe burn injury.

Our study wished to address whether there is firstly consistency in outcome reporting and secondly whether studies report burns-specific patient-centred outcomes. To answer this, we applied two separate frameworks. Given the uptake of the OMERACT domains for COS development in other critical care fields, we chose to classify outcomes according to the framework as a means of assessing the consistency of outcome reporting. To assess if trials report burns-specific patient outcomes, we applied a framework proposed by Falder et al that assesses crucial long term outcomes in burns survivors [12]. The framework assesses patients' skin, neuromuscular function, somatosensory perception (pain, itch), psychological function, physical role function, community participation and perceived quality of life.

\section{Methods}

The study was conducted according to a pre-specified protocol (see Appendix C), in alignment with the PRISMA guidelines and checklist on systematic review design [13].

\section{Study eligibility}

The study included randomised clinical trials (RCTs), pseudo-randomised clinical trials, comparative studies with concurrent controls, and intervention studies without concurrent controls that investigated adult burns patients managed in the ICU. Studies were deemed as pseudo-randomised if patients were assigned to a study arm by alternate allocation rather than true randomisation [14]. Studies were included only if the intervention was deemed a key component of severe burns management (as per our pre-specified protocol). These interventions included fluid resuscitation, transfusion strategy, ventilation strategy, nutrition, analgesia, haemodynamic monitoring or timing of surgery. Studies were included if they were written in English, enrolled human subjects and a primarily adult population. Systematic reviews, meta-analyses and case series were not included.

\section{Data search}

We conducted a literature search through PubMed and Medline (via Ovid), using MeSH terms for burns and intensive care, and the domains listed above.

The search strategy for the study was:

(()((()(isotonic solution OR crystalloid OR saline OR intravenous fluid)) OR (analgesia OR anaesthesia and analgesia OR pain management)) OR physiologic 
monitoring) OR (pulmonary ventilation OR invasive ventilation OR non-invasive ventilation)) OR (blood transfusion OR blood product transfusion OR transfusion)) OR enteral nutrition) AND (burns OR thermal injury OR burns injury OR chemical injury OR electrical injury)) AND (intensive care OR critical care OR intensive care unit OR critically ill OR critical illness)

The time frame for the search was limited to studies published between January 1, 1960, and December 31, 2019.

\section{Data collection}

Each study was reviewed by two authors to ensure consistency in data collection. We documented the following information about each study: first author, year of publication, type of study (RCT, pseudo-RCT, comparative study with concurrent controls or intervention study without concurrent controls), patient population (degree of burn injury and salient inclusion/exclusion criteria), intervention and control, as well as the primary outcome and longest documented follow-up. If the longest follow-up was not reported, we attempted to derive it by taking the longest reported outcome.

\section{Outcome classification}

Primary outcomes were reviewed and classified as either patient centred or surrogate outcomes $[15,16]$. Patientcentred outcomes were defined as those deemed relevant to patients in both the short and long term. Examples of these include mortality, measures of quality of life (e.g. psychological function, functionality, independence), pain (acute or chronic), adverse outcomes from therapy, duration of mechanical ventilation and ICU/hospital length of stay (LOS). Surrogate outcomes included biomarkers, vital signs, radiological or histological findings and other markers that were not perceived to correlate with patients' quality of life. In addition, when classifying outcomes with the OMERACT and Falder frameworks, both primary and secondary outcomes were reviewed.

For the OMERACT outcome classification, we tabulated whether each study reported outcomes relevant to each domain in their results or discussion sections. The data was recorded as whether an outcome relevant to the domain was reported or not. Examples of outcome measures classified into each domain were: Mortality (was death reported as an outcome Yes/No), Life Impact (was a patient-centred outcome, either short or longterm, reported?), Pathophysiological Manifestation (biomarkers, clinical manifestations, vital signs) and Resource Use (direct measurement of costs or surrogate markers of cost including ICU length of stay, hospital length of stay).
For the burns-specific outcomes listed by Falder, we recorded whether each study documented an outcome relevant to any of the seven domains listed in the framework above. The data was reported as a Yes/No whether an appropriate outcome was reported.

\section{Data synthesis}

Quantitative and qualitative data from the studies was derived and tabulated with counts and proportions reported. To present both the OMERACT and Falder outcomes, data was broken down into intervention subheadings, and the number of studies reporting each domain presented as absolute numbers and percentages. The total number of papers and percentages for each framework domain were also calculated and presented in the tabulated data.

\section{Results}

A total 6154 studies were initially identified, with 98 papers meeting inclusion criteria (see PRISMA flow diagram in Fig. 1). There were 19 studies on analgesia, 26 studies on fluid resuscitation, 4 on haemodynamic monitoring, 31 on nutrition, 5 on surgical timing, 8 on transfusion strategies and 5 on ventilation strategies.

\section{Trial characteristics}

Trial characteristics and overall findings per trial type are listed in detail in Table 1 (see below-findings expressed as absolute numbers and percentages). Detailed tables with outcomes for each study have been included in the Appendix (Appendix A: Tables 2-10). The search returned 53 RCTs, 13 pseudo-RCTs, 19 clinical studies with concurrent controls and 13 intervention studies without concurrent controls. Across all studies, the median number of patients per trial was 40 (IQR 24-60), with only 13 (13\%) enrolling greater than 100 . Seven studies (7\%) were conducted across more than one centre while the remainder were single centre studies. Median longest follow-up (LFU) was low across all study types. The type of study did not appear to affect the frequency and consistency by which OMERACT or Falder outcomes were reported. Findings for each study domain are reported in greater detail subsequently.

\section{Analgesia}

The results of the analgesia studies are listed in Table 2 in Appendix A. In all except one study, the primary outcome measured was pain. However, three different pain scales were used with the visual analogue scale (VAS) the most commonly applied (83\%). One study (5\%) did not report a patient- 


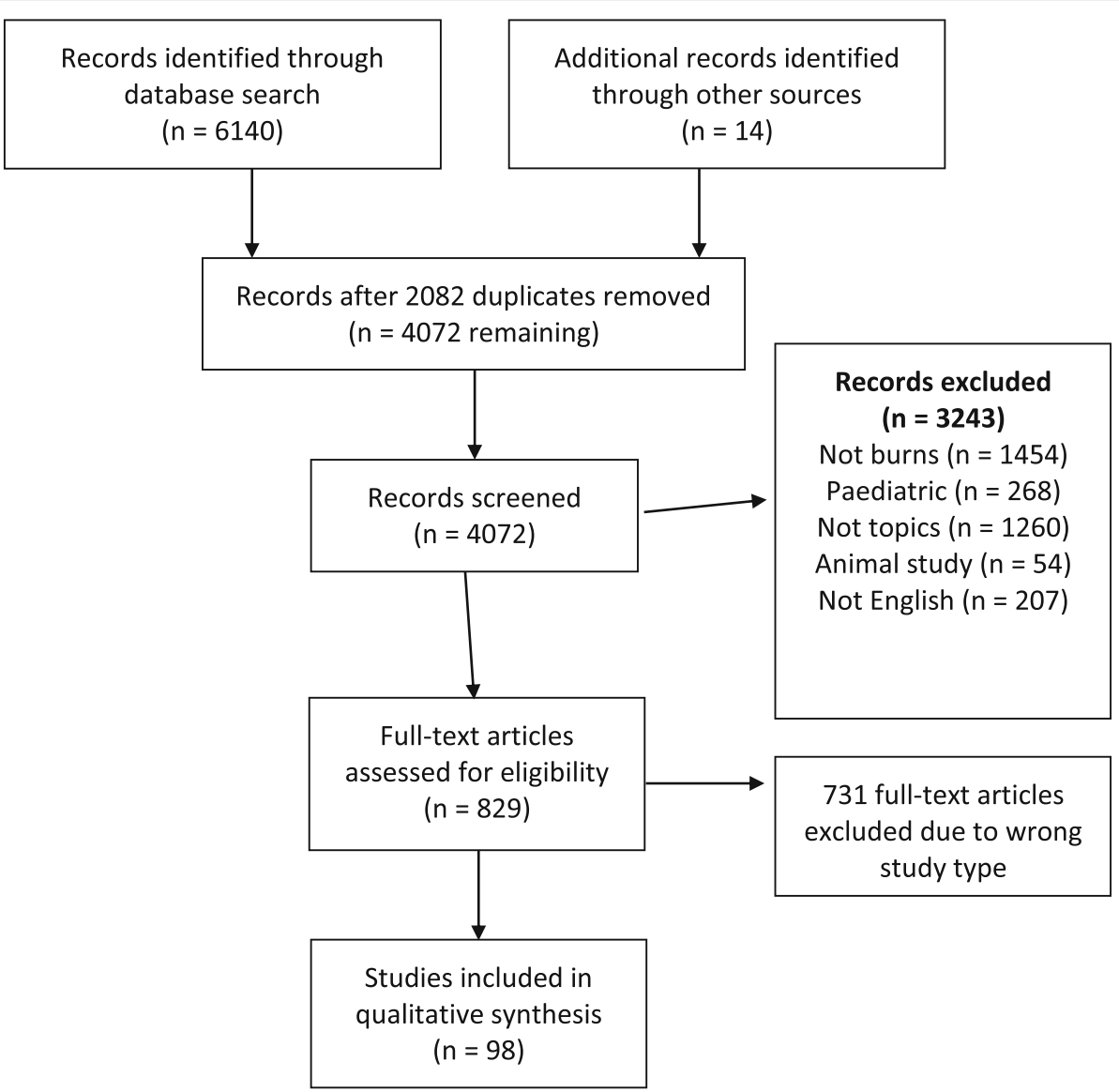

Fig. 1 PRISMA flow diagram of study exclusion and inclusion into systematic review

centred primary outcome; this was the study by Promes et al, which assessed area under the curve for patient temperature as a primary outcome. Median longest follow-up was 2 days (IQR 1 to 7 ). Only four studies (20\%) had a follow-up greater than 14days and only 1 study assessed pain at 6 months.
Fluid resuscitation

The results of the fluid resuscitation studies are listed in Table 3 in Appendix A. The primary outcome in nine studies (35\%) was fluid volume administered. In the remaining 17 studies, there were 13 different primary outcomes. Only fluid balance, urine

Table 1 Findings broken down by study type

\begin{tabular}{|c|c|c|c|c|c|c|c|c|c|}
\hline \multirow[t]{2}{*}{ Study type } & \multirow{2}{*}{$\begin{array}{l}\text { No. of } \\
\text { studies }\end{array}$} & \multirow{2}{*}{$\begin{array}{l}\text { Median } \\
\text { no. of } \\
\text { patients }\end{array}$} & \multirow{2}{*}{$\begin{array}{l}\text { Patient- } \\
\text { centred } \\
\text { primary } \\
\text { outcome, } \\
n(\%)\end{array}$} & \multirow{2}{*}{$\begin{array}{l}\text { Median } \\
\text { LFU } \\
\text { (days) }\end{array}$} & \multicolumn{4}{|c|}{ OMERACT, $n$ (\%) } & \multirow{2}{*}{$\begin{array}{l}\text { Falder } \\
\text { outcome, } \\
n(\%)\end{array}$} \\
\hline & & & & & Mortality & Life impact & Resource/economic & Pathophys. & \\
\hline$\overline{\mathrm{RCT}}$ & 53 & 43 & $20(37)$ & 3 & $24(45)$ & $18(33)$ & $13(25)$ & $41(77)$ & $17(32)$ \\
\hline Pseudo-RCT & 13 & 31 & $3(23)$ & 7 & $9(69)$ & $0(0)$ & $4(30)$ & $12(92)$ & $2(15)$ \\
\hline CSWCC & 19 & 30 & $6(31)$ & 28 & $11(58)$ & $6(32)$ & $5(26)$ & $14(74)$ & $5(26)$ \\
\hline ISWCC & 13 & 40 & $3(23)$ & 12 & $10(77)$ & $3(23)$ & $8(62)$ & $11(85)$ & $2(15)$ \\
\hline
\end{tabular}

$R C T$ randomised control trial, Pseudo-RCT Pseudo-randomised control trial, CSWCC clinical study with concurrent control, ISWCC intervention study without concurrent control, LFU longest follow-up 
output, cardiac output and multiple organ dysfunction score (MODS) were common primary outcomes. Only two studies (7\%) reported a patient centredoutcome. Median longest follow-up was 3 days (IQR 2 to 28). Longest follow-up was not available in two studies.

\section{Haemodynamic monitoring}

The results of the haemodynamic monitoring studies are listed in Table 4 in Appendix A. There was no consistency in the primary outcomes measured in all four studies, three $(75 \%)$ of which were surrogate outcomes. Median longest follow-up was 3 days (IQR 3-37).

\section{Nutrition}

The results of the nutrition studies are listed in Table 5 in Appendix A. There were 26 different primary outcomes across the 31 studies; 25 of these $(81 \%)$ were surrogate outcomes. Only four of these outcomes were common to more than one study with nitrogen balance the most frequent measure (three studies). Median longest follow-up was 6 days (IQR 3-28). Longest follow-up was not available in three studies.

\section{Surgical timing}

The results of the surgical studies are listed in Table 6 in Appendix A. Mortality was the primary outcome in two of the studies. The remaining three studies had differing primary outcomes, all of which were surrogate measures. Median longest follow-up was 42 days (IQR 30-180). One study reported follow-up at 6 months and one study did not report follow -up.

\section{Transfusion strategies}

The results of the transfusion studies are listed in Table 7 in Appendix A. Transfusion requirement and haemoglobin concentration pre- and post-operative were the primary outcome for two studies each. The remaining four studies had different outcome measures. Only one study reported a patient-centred primary outcome. Median longest follow-up was 25 days (IQR 11-30). Followup was not reported in one study.

\section{Ventilation strategies}

The results of the ventilation studies are listed in Table 8 in Appendix A. Of the five studies, two had the same primary outcome (lung injury score). Two primary outcomes were patient-centred (assessing duration of mechanical ventilation). Median longest follow-up was 18 days (IQR 6-33).
OMERACT and Falder outcome classification reporting

Tables 9 and 10 in Appendix A summarise the outcome classification for the papers found in this systematic review according to the OMERACT and Falder frameworks. Regarding the OMERACT classification, in summary, $53 \%$ of papers reported mortality as an outcome, $28 \%$ reported outcomes related to life impact, $30 \%$ reported on resource and economic use and 95\% reported on outcomes related to pathophysiological manifestations. Pathophysiological manifestations were consistently reported across all domains; the outcomes were predominantly haemodynamic parameters, measurements of organ function and biochemical values and biomarkers. Economic-related outcomes were mainly surrogate outcomes of cost (ICU or hospital LOS), although one paper (Saffle et al.) measured hospital costs as an outcome. The majority of the life impact outcomes were in the analgesia studies, where all 19 studies reported pain as an outcome.

The burns specific outcome set proposed by Falder et al was poorly adhered to. Across the 98 papers only 29 times out of a possible 686 occasions did the outcome apply to one the suggested domains, equating to a reporting rate of $4.4 \%$. Nearly all of these were reported in the analgesia studies, with all reporting pain, and only two studies reporting psychological function and 1 study reporting quality of life. The surgical timing study by Puri et al. was the only study with intention to assess neuromuscular function however it could not be completed due to poor follow-up.

There did not appear to be a trend in improved patient-centred outcome reporting with more recent studies compared to older studies.

\section{Discussion}

This systematic review was undertaken to assess the outcomes reported in the literature on management of severe burns patients from January 1960 to December 2019. We reviewed studies that addressed the seven fields of care that are fundamental to the management of burns patients in the acute care of severe burns.

Our study has highlighted deficiencies in outcome reporting in acute burn care literature. The outcomes measured are highly variable, at inconsistent time frames (usually short) and are rarely meaningful, patient-centred end points. Application of the OMERACT framework has demonstrated that studies of critically ill patients with severe burns patients only consistently report pathophysiological manifestations; however, the specific outcomes are highly variable. Even on review of the RCTs or pseudo-RCTs (which should theoretically be well-designed studies with pre- 
specified outcomes), there did not appear to be any consistency in outcomes when the OMERACT framework was applied. Reporting of patient-centred outcomes overall was poor. Our study has demonstrated that only the analgesia study subgroup consistently reported these outcomes; however, it is important to note that this was limited almost only to pain with short time end points. Survivors of severe burn injury are at high risk of chronic pain and given the current opioid epidemic, studies of analgesia should ideally look at long-term pain outcomes.

While the initial focus of burn care in the ICU is resuscitation and prevention of complications, the improved rates of burn survival mandate that studies of severe burn injury look at long term outcomes. We have demonstrated a low rate of burns specific outcome reporting (from the Falder framework), and moving forward, it is important to establish how early interventions are impacting on patients' day-to-day lives when they are discharged from hospital and return to the community.

The absence of consistent, meaningful outcome reporting in the acute burns literature impacts the overall quality of the evidence and limits the ability to use it to guide clinical practice. A recent survey of Australian and New Zealand burns intensive care units demonstrated marked variability in practices [17], which likely reflects the quality status of the burns literature. Standardisation of outcome reporting would allow better comparison between burn units and help to identify areas of variable outcomes. These could then be the focus of research to determine what constitutes best practice and ultimately lead to improved patient outcomes.

Systematic reviews of other domains of intensive care have demonstrated inconsistent outcome selection and timing of outcome reporting between trials, which has hindered the development of guidelines and recommendations [11]. Further this to, with improving survival in intensive care units, there is a push to investigate outcomes beyond mortality and assess long-term patient-centred outcomes including morbidity, functionality and mental health [18]. The establishment of COS in critical care research has become a focus within the last decade. The COMET (Core Outcome Measures in Effectiveness Trials) initiative and the InFACT (International forum for acute care trialists) initiative have been instrumental in developing COS in cardiac arrest [19] and acute respiratory failure [20]. Studies are currently underway to establish $\operatorname{COS}$ in other important critical care domains including aneurysmal subarachnoid haemorrhage [21], physical rehabilitation [22] and delirium [23].

\section{Strengths and limitations}

This is the first systematic review of the critical care burns literature that has investigated core outcome reporting, spanning six decades of clinical research. By including a variety of trial designs in our search, we have aimed to encompass a large body of the literature relevant to critically ill burns patients. While there are obvious differences between rheumatological diseases and acute burns, we applied the OMERACT framework in our study as it is well validated and has a broad set of domains that have previously guided COS development in other specialities [24, 25]. The outcome set proposed by Falder is specific to burns patients; however, recording that dataset requires a two-hour patient interview so may not meet the standard for feasibility. We excluded studies not written in English and therefore may have omitted studies from developing countries, which may have a higher incidence of burn injury and therefore valuable data. While our search strategy was limited to Medline (Ovid) and PubMed, the content within these two databases should be representative of the vast majority of high quality scientific evidence.

\section{Recommendations}

Based on the findings of this review, we recommend that a clinically relevant COS is created which can be applied to future burns research. Development of a COS will require multidisciplinary consensus input from burn care specialists, surgeons, intensivists, anaesthetists, rehabilitation specialists and allied health workers. Support from the COMET and InFACT initiatives would aid this process. Given that severe burns occur with higher frequency in developing countries, it would be important that development of a COS takes into account limitations of resource-poor nations.

\section{Conclusion}

This study has demonstrated that outcome reporting in the literature related to severe burns patients in the ICU is highly variable, rarely patient-centred and with a lack of long term follow-up. The development of an accepted and validated core outcome dataset that encompasses outcomes meaningful to our patients would improve the quality and standardisation of outcome reporting. This would lead to improvement in the quality of the burns literature, and eventually improved care and patient outcomes. 


\section{Appendix A}

Table 2 Analgesia studies

\begin{tabular}{|c|c|c|c|c|c|c|c|c|c|c|}
\hline Author & Year & Study & Centre(s) & Number & Population & Intervention & Control & $\begin{array}{l}\text { Primary } \\
\text { outcome }\end{array}$ & $\begin{array}{l}\text { Surrogate } \\
\text { vs patient- } \\
\text { centred }\end{array}$ & $\begin{array}{l}\text { LFU } \\
\text { (days) }\end{array}$ \\
\hline $\begin{array}{l}\text { Choiniere } \\
\text { et al. [26] }\end{array}$ & 1992 & $\mathrm{RCT}$ & 1 & 24 & TBSA $>15 \%$ & Morphine PCA & $\begin{array}{l}\text { Nurse administered PRN IV } \\
\text { morphine }\end{array}$ & Pain per VAS & $\begin{array}{l}\text { Patient- } \\
\text { centred }\end{array}$ & 3 \\
\hline $\begin{array}{l}\text { Cuignet et al. } \\
{[27]}\end{array}$ & 2004 & $\mathrm{RCT}$ & 1 & 20 & $\begin{array}{l}\text { TBSA }>15 \% \text {, } \\
\text { undergoing skin graft } \\
\text { surgery }\end{array}$ & $\begin{array}{l}\text { Ropivacaine fascia iliaca block } \\
\text { to donor site }\end{array}$ & $\begin{array}{l}0.9 \% \text { saline fascia iliaca } \\
\text { infusion }\end{array}$ & Pain per VAS & $\begin{array}{l}\text { Patient- } \\
\text { centred }\end{array}$ & 3 \\
\hline $\begin{array}{l}\text { Everett et al. } \\
{[28]}\end{array}$ & 1993 & $\mathrm{RCT}$ & 1 & 32 & $\begin{array}{l}\text { Burn injuries requiring } \\
>4 \text { days hospitalisation } \\
\text { and debridement. }\end{array}$ & $\begin{array}{l}\text { Hypnosis } \pm \text { lorazepam in } \\
\text { addition to opioids }\end{array}$ & Opioids & Pain per VAS & $\begin{array}{l}\text { Patient- } \\
\text { centred }\end{array}$ & 4 \\
\hline Finn et al. [29] & 2004 & $\mathrm{RCT}$ & 1 & 26 & $\begin{array}{l}\text { Age } \geq 18 \text { years, } \\
\text { requiring dressing } \\
\text { change } \pm \text { debridement }\end{array}$ & $\begin{array}{l}\text { Patient controlled intra-nasal } \\
\text { fentanyl }\end{array}$ & Oral morphine & Pain per NRS & $\begin{array}{l}\text { Patient- } \\
\text { centred }\end{array}$ & 2 \\
\hline Gray et al. [30] & 2011 & $\mathrm{RCT}$ & 1 & 121 & $\begin{array}{l}\text { TBSA } \geq 5 \% \text { of any } \\
\text { depth requiring } \\
\text { admission to the burn } \\
\text { unit }\end{array}$ & Pregabalin & Placebo & Pain per NRS & $\begin{array}{l}\text { Patient- } \\
\text { centred }\end{array}$ & $\begin{array}{l}180 \\
\text { days (6 } \\
\text { months) }\end{array}$ \\
\hline $\begin{array}{l}\text { Gunduz et al. } \\
\text { [31] }\end{array}$ & 2011 & $\mathrm{RCT}$ & 1 & 90 & $\begin{array}{l}\text { TBSA } 10-25 \% \\
\text { undergoing dressing } \\
\text { changes }\end{array}$ & $\begin{array}{l}\text { Midazolam/dexmedetomidine } \\
\text { added to analgesic/sedative } \\
\text { regime for dressing changes }\end{array}$ & Ketamine & Pain per VAS & $\begin{array}{l}\text { Patient- } \\
\text { centred }\end{array}$ & $<1$ \\
\hline $\begin{array}{l}\text { Jellish et al. } \\
\text { [32] }\end{array}$ & 1999 & $\mathrm{RCT}$ & 1 & 60 & TBSA $>10 \%$ & $\begin{array}{l}\text { Aerosolised } 2 \% \text { lidocaine } w / 1 \text { : } \\
200,000 \text { adrenaline to graft } \\
\text { donor site }\end{array}$ & $\begin{array}{l}0.9 \% \text { NS w/ 1:200,000 } \\
\text { adrenaline OR 0.5\% } \\
\text { bupivacaine w/ 1:200,000 } \\
\text { adrenaline }\end{array}$ & Pain per VAS & $\begin{array}{l}\text { Patient- } \\
\text { centred }\end{array}$ & 2 \\
\hline $\begin{array}{l}\text { Kundra et al. } \\
\text { [33] }\end{array}$ & 2013 & $\mathrm{RCT}$ & 1 & 60 & $\begin{array}{l}\text { TBSA }>35 \% \text { undergoing } \\
\text { wound dressing } \\
\text { changes }\end{array}$ & Oral ketamine & Oral dexmedetomidine & Pain per VAS & $\begin{array}{l}\text { Patient- } \\
\text { centred }\end{array}$ & 2 \\
\hline Lee et al. [34] & 1989 & $\mathrm{RCT}$ & 1 & 50 & $\begin{array}{l}\text { TBSA }>10 \% \text { undergoing } \\
\text { burn wound } \\
\text { debridement }\end{array}$ & IV nalbuphine hydrochloride & Intravenous morphine & Pain per VAS & $\begin{array}{l}\text { Patient- } \\
\text { centred }\end{array}$ & 2 \\
\hline $\begin{array}{l}\text { Patterson } \\
\text { et al. [35] }\end{array}$ & 1997 & $\mathrm{RCT}$ & 1 & 79 & $\begin{array}{l}\text { TBSA > 15\% requiring } \\
\text { wound debridement. }\end{array}$ & $\begin{array}{l}\text { Lorazepam in addition to } \\
\text { opioids }\end{array}$ & Opioids & Pain per VAS & $\begin{array}{l}\text { Patient- } \\
\text { centred }\end{array}$ & 4 \\
\hline $\begin{array}{l}\text { Prakash et al. } \\
\text { [36] }\end{array}$ & 2004 & $\mathrm{RCT}$ & 1 & 60 & $\begin{array}{l}\text { TBSA }>20 \% \text {, able to use } \\
\text { a PCA during dressing } \\
\text { changes }\end{array}$ & Fentanyl PCA & $\begin{array}{l}\text { Nil placebo or specific } \\
\text { control }\end{array}$ & Pain per VAS & $\begin{array}{l}\text { Patient- } \\
\text { centred }\end{array}$ & 1 \\
\hline Raza et al. [37] & 2014 & $\mathrm{RCT}$ & 1 & 150 & $\begin{array}{l}\text { Undergoing split skin } \\
\text { grafts with dressing } \\
\text { changes }\end{array}$ & $\begin{array}{l}\text { Bupivacaine-soaked gauze to } \\
\text { donor sites }\end{array}$ & $\begin{array}{l}\text { Saline-soaked gauze to } \\
\text { donor sites }\end{array}$ & Pain per VAS & $\begin{array}{l}\text { Patient- } \\
\text { centred }\end{array}$ & 1 \\
\hline $\begin{array}{l}\text { Wasiak et al. } \\
\text { [38] }\end{array}$ & 2011 & $\mathrm{RCT}$ & 1 & 45 & $\begin{array}{l}\text { TBSA }>10 \% \\
\text { undergoing dressing } \\
\text { changes }\end{array}$ & $\begin{array}{l}\text { IV lidocaine for analgesia in } \\
\text { addition to usual morphine } \\
\text { PCA }\end{array}$ & $\begin{array}{l}\text { IV placebo with usual } \\
\text { morphine PCA }\end{array}$ & Pain per VRS & $\begin{array}{l}\text { Patient- } \\
\text { centred }\end{array}$ & 2 \\
\hline $\begin{array}{l}\text { Wibbenmeyer } \\
\text { et al. [39] }\end{array}$ & 2014 & $\mathrm{RCT}$ & 1 & 53 & $\begin{array}{l}>5 \% \text { TBSA, expected } \\
\text { LOS }>48 \mathrm{~h}\end{array}$ & Gabapentin & Placebo & $\begin{array}{l}\text { Morphine } \\
\text { consumption }\end{array}$ & Surrogate & $\begin{array}{l}43 \text { days } \\
\text { post D/ } \\
\text { C }\end{array}$ \\
\hline $\begin{array}{l}\text { Yuxiang et al. } \\
\text { [40] }\end{array}$ & 2012 & $\mathrm{RCT}$ & 3 & 240 & $\begin{array}{l}1-70 \% \text { TBSA requiring } \\
\text { dressing change }\end{array}$ & $\begin{array}{l}\text { Inhaled nitrous oxide added to } \\
\text { analgesia }\end{array}$ & $\begin{array}{l}\text { Analgesia plus inhaled } \\
\text { oxygen }\end{array}$ & Pain per VAS & $\begin{array}{l}\text { Patient- } \\
\text { centred }\end{array}$ & $<1$ \\
\hline Zor et al. [41] & 2010 & $\mathrm{RCT}$ & 1 & 24 & TBSA $20-50 \%$ & $\begin{array}{l}\text { IM ketamine or } \\
\text { dexmedetomidine. or } \\
\text { midazolam in addition to usual } \\
\text { analgesia }\end{array}$ & $\begin{array}{l}\text { Standard care for } \\
\text { procedural pain-ketamine } \\
\text { alone (group I) }\end{array}$ & Pain per VAS & $\begin{array}{l}\text { Patient- } \\
\text { centred }\end{array}$ & 10 \\
\hline $\begin{array}{l}\text { Foertsch et al. } \\
\text { [42] }\end{array}$ & 1995 & $\begin{array}{l}\text { CSWC } \\
C\end{array}$ & 2 & 106 & TBSA > 15\% & Morphine & No morphine & Pain per VAS & $\begin{array}{l}\text { Patient- } \\
\text { centred }\end{array}$ & 65 \\
\hline $\begin{array}{l}\text { Nilsson et al. } \\
\text { [43] }\end{array}$ & 2008 & $\begin{array}{l}\text { CSWC } \\
C\end{array}$ & 1 & 11 & $\begin{array}{l}\text { TBSA }>10 \% \text { undergoing } \\
\text { dressing changes }\end{array}$ & $\begin{array}{l}\text { Patient controlled sedation } \\
\text { (propofol } 20 \mathrm{mg} / \mathrm{ml} \text { and } \\
\text { alfentanil } 0.13 \mathrm{mg} / \mathrm{ml} \text { ) }\end{array}$ & $\begin{array}{l}\text { Anaesthetist led sedation } \\
\text { (propofol } 10 \mathrm{mg} / \mathrm{ml} \text { and } \\
\text { fentanyl } 50 \mathrm{mcg} / \mathrm{ml} \text { ) }\end{array}$ & Pain per VAS & $\begin{array}{l}\text { Patient- } \\
\text { centred }\end{array}$ & 1 \\
\hline $\begin{array}{l}\text { Berger et al. } \\
{[44]}\end{array}$ & 2010 & $\begin{array}{l}\text { ISWC } \\
C\end{array}$ & 1 & 46 & TBSA not specified & $\begin{array}{l}\text { Hypnosis in conjunction with } \\
\text { pharmacological analgesia }\end{array}$ & Pharmacological analgesia & Pain per VAS & $\begin{array}{l}\text { Patient- } \\
\text { centred }\end{array}$ & 40 \\
\hline
\end{tabular}

$R C T$ randomised control trial, CSWCC clinical study with concurrent control, ISWCC intervention study without concurrent control, TBSA total burn surface area, VAS visual analogue scale, NRS numeric rating scale, VRS verbal rating scale, LFU longest follow-up 
Table 3 Fluid resuscitation studies

\begin{tabular}{|c|c|c|c|c|c|c|c|c|c|c|}
\hline Author & Year & $\begin{array}{l}\text { Study } \\
\text { type }\end{array}$ & Centre(s) & Number & Population & Intervention & Control & $\begin{array}{l}\text { Primary } \\
\text { outcome }\end{array}$ & $\begin{array}{l}\text { Surrogate } \\
\text { vs } \\
\text { patient- } \\
\text { centred }\end{array}$ & $\begin{array}{l}\text { LFU } \\
\text { (days) }\end{array}$ \\
\hline $\begin{array}{l}\text { Bechir } \\
\text { et al. [45] }\end{array}$ & 2013 & $\mathrm{RCT}$ & 1 & 48 & TBSA > 15\% & $\begin{array}{l}\text { Hydroxyethyl } \\
\text { starch with RL }\end{array}$ & $\mathrm{RL}$ & $\begin{array}{l}\text { Fluid volume } \\
\text { administered }\end{array}$ & Surrogate & 28 \\
\hline $\begin{array}{l}\text { Bedi et al. } \\
\text { [46] }\end{array}$ & 2019 & $\mathrm{RCT}$ & 1 & 200 & TBSA $>30 \%$ & $\begin{array}{l}\text { Dextrose }+ \\
0.9 \% \text { normal } \\
\text { saline }\end{array}$ & $\mathrm{RL}$ & Serum sodium & Surrogate & 3 \\
\hline $\begin{array}{l}\text { Belba et al. } \\
{[47]}\end{array}$ & 2009 & $\mathrm{RCT}$ & 1 & 110 & $\begin{array}{l}\text { TBSA }>20 \% \text { adults, }> \\
15 \% \text { children }\end{array}$ & $\begin{array}{l}\text { Hypertonic } \\
\text { lactate saline }\end{array}$ & $\mathrm{RL}$ & $\begin{array}{l}\text { Cumulative fluid } \\
\text { balance }\end{array}$ & Surrogate & 1 \\
\hline $\begin{array}{l}\text { Bortolani } \\
\text { et al. [48] }\end{array}$ & 1996 & $\mathrm{RCT}$ & 1 & 40 & TBSA $>30 \%$ & $\begin{array}{l}\text { Hypertonic } \\
\text { lactate saline }\end{array}$ & $\mathrm{RL}$ & $\begin{array}{l}\text { Fluid volumes } \\
\text { administered }\end{array}$ & Surrogate & 4 \\
\hline $\begin{array}{l}\text { Cooper } \\
\text { et al. [49] }\end{array}$ & 2006 & $\mathrm{RCT}$ & 3 & 42 & TBSA $>20 \%$ & $5 \%$ albumin & $\mathrm{RL}$ & $\begin{array}{l}\text { Difference in } \\
\text { MODS between } \\
\text { groups }\end{array}$ & Surrogate & 28 \\
\hline $\begin{array}{l}\text { Goodwin } \\
\text { et al. [50] }\end{array}$ & 1983 & $\mathrm{RCT}$ & 1 & 79 & TBSA unknown & $\begin{array}{l}\text { Albumin- } \\
\text { Ringer's } \\
\text { solution }\end{array}$ & $\mathrm{RL}$ & Cardiac output & Surrogate & 7 \\
\hline $\begin{array}{l}\text { Gunn et al. } \\
\text { [51] }\end{array}$ & 1989 & $\mathrm{RCT}$ & 1 & 51 & $>20 \%$ TBSA & $\begin{array}{l}\text { Hypertonic } \\
\text { lactate saline }\end{array}$ & $\mathrm{RL}$ & $\begin{array}{l}\text { Fluid volume } \\
\text { administered }\end{array}$ & Surrogate & 3 \\
\hline $\begin{array}{l}\text { Hall et al. } \\
{[52]}\end{array}$ & 1978 & $\mathrm{RCT}$ & 1 & 172 & $\begin{array}{l}\text { TBSA }>15 \% \text { adults, }> \\
10 \% \text { children }\end{array}$ & Dextran 70 & $\mathrm{RL}$ & Urine output & Surrogate & 3 \\
\hline $\begin{array}{l}\text { Huang } \\
\text { et al. [53] }\end{array}$ & 2005 & $\mathrm{RCT}$ & 1 & 20 & TBSA $>40 \%$ & $\begin{array}{l}\text { Delayed rapid } \\
\text { colloid } \\
\text { resuscitation }\end{array}$ & $\begin{array}{l}\text { No rapid } \\
\text { fluid } \\
\text { resuscitation }\end{array}$ & $\begin{array}{l}\text { Fluid volume } \\
\text { administered }\end{array}$ & Surrogate & 2 \\
\hline $\begin{array}{l}\text { Sudhakar } \\
\text { et al. [54] }\end{array}$ & 2008 & $\mathrm{RCT}$ & 1 & 32 & TBSA 30-70\% & $\begin{array}{l}\text { Hydroxyethyl } \\
\text { starch 130/0.4 } \\
+ \text { RL }\end{array}$ & $\mathrm{RL}$ & Urine output & Surrogate & 2 \\
\hline $\begin{array}{l}\text { Vlachou } \\
\text { et al. [55] }\end{array}$ & 2010 & $\mathrm{RCT}$ & 1 & 26 & TBSA $15-80 \%$ & $\begin{array}{l}6 \% \\
\text { hydroxyethyl } \\
\text { starch + RL }\end{array}$ & $\mathrm{RL}$ & Fluid balance & Surrogate & 2 \\
\hline $\begin{array}{l}\text { Waxman } \\
\text { et al. [56] }\end{array}$ & 1989 & $\mathrm{RCT}$ & 1 & 12 & TBSA $>25 \%$ & $\begin{array}{l}10 \% \\
\text { pentastarch }\end{array}$ & $5 \%$ albumin & $\begin{array}{l}\text { Haemodynamic } \\
\text { parameters }\end{array}$ & Surrogate & $<1$ \\
\hline $\begin{array}{l}\text { Aoki et al. } \\
\text { [57] }\end{array}$ & 2010 & $\begin{array}{l}\text { Pseudo } \\
\text { RCT }\end{array}$ & 2 & 20 & TBSA $>30 \%$ & RA & $\mathrm{RL}$ & Gastric $\mathrm{CO}_{2}$ & Surrogate & 3 \\
\hline $\begin{array}{l}\text { O'mara } \\
\text { et al. [58] }\end{array}$ & 2005 & $\begin{array}{l}\text { Pseudo } \\
\text { RCT }\end{array}$ & 1 & 31 & $\begin{array}{l}\text { TBSA > 40\% without } \\
\text { inhalational injury OR } \\
\text { TBSA > 25\% with } \\
\text { inhalational injury }\end{array}$ & $\mathrm{RL}$ and FFP & $\mathrm{RL}$ & IAP $>25 \mathrm{mmHg}$ & Surrogate & 5 \\
\hline $\begin{array}{l}\text { Tanaka } \\
\text { et al. [59] }\end{array}$ & 2000 & $\begin{array}{l}\text { Pseudo } \\
\text { RCT }\end{array}$ & 1 & 37 & TBSA $>30 \%$ & $\begin{array}{l}\text { IV ascorbic } \\
\text { acid + RL }\end{array}$ & $\mathrm{RL}$ & $\begin{array}{l}\text { Fluid volume } \\
\text { administered }\end{array}$ & Surrogate & 36 \\
\hline $\begin{array}{l}\text { Bechir } \\
\text { et al. [60] }\end{array}$ & 2010 & CSWCC & 1 & 30 & TBSA unknown & $\begin{array}{l}\text { Hydroxyethyl } \\
\text { starch + RL }\end{array}$ & $\mathrm{RL}$ & $\begin{array}{l}\text { Fluid volume } \\
\text { administered }\end{array}$ & Surrogate & 60 \\
\hline $\begin{array}{l}\text { Bocanegra } \\
\text { et al. [61] }\end{array}$ & 1966 & CSWCC & 1 & 308 & TBSA $>10 \%$ & $\begin{array}{l}\text { Colloid-plus- } \\
\text { glucose or } \\
\text { saline-plus- } \\
\text { plasma }\end{array}$ & NS & Shock mortality & $\begin{array}{l}\text { Patient- } \\
\text { centred }\end{array}$ & 36 \\
\hline $\begin{array}{l}\text { Chung } \\
\text { et al. [62] }\end{array}$ & 2009 & CSWCC & 1 & 52 & TBSA $>20 \%$ & $\begin{array}{l}\text { Brooke } \\
\text { formula }\end{array}$ & $\begin{array}{l}\text { Parkland } \\
\text { formula }\end{array}$ & $\begin{array}{l}\text { Fluid volume } \\
\text { administered }\end{array}$ & Surrogate & 1 \\
\hline $\begin{array}{l}\text { Jelenko } \\
\text { et al. [63] }\end{array}$ & 1978 & CSWCC & 1 & 19 & TBSA $>40 \%$ & $\begin{array}{l}\text { Hypertonic } \\
\text { albumin } \\
\text { solution }\end{array}$ & $\begin{array}{l}2 \text { groups- } \\
\text { (A) } R L,(H) \\
\text { hypertonic } \\
\text { solution }\end{array}$ & Weight change & Surrogate & 5 \\
\hline $\begin{array}{l}\text { Murphy } \\
\text { et al. [64] }\end{array}$ & 1999 & CSWCC & 1 & 18 & $\mathrm{TBSA}>40 \%$ & $\begin{array}{l}\mathrm{RL} \text { and } 7.5 \% \\
\text { hypertonic } \\
\text { saline-dextran } \\
\text { solution }\end{array}$ & $\begin{array}{l}\text { Ringer's } \\
\text { lactate only }\end{array}$ & $\begin{array}{l}\text { Cardiac output } \\
\text { parameters as } \\
\text { measured by PA } \\
\text { catheter }\end{array}$ & Surrogate & 1 \\
\hline
\end{tabular}


Table 3 Fluid resuscitation studies (Continued)

\begin{tabular}{|c|c|c|c|c|c|c|c|c|c|c|}
\hline Author & Year & $\begin{array}{l}\text { Study } \\
\text { type }\end{array}$ & Centre(s) & Number & Population & Intervention & Control & $\begin{array}{l}\text { Primary } \\
\text { outcome }\end{array}$ & $\begin{array}{l}\text { Surrogate } \\
\text { vs } \\
\text { patient- } \\
\text { centred }\end{array}$ & $\begin{array}{l}\text { LFU } \\
\text { (days) }\end{array}$ \\
\hline $\begin{array}{l}\text { Oda et al. } \\
\text { [65] }\end{array}$ & 2006 & CSWCC & 1 & 36 & TBSA $>40 \%$ & $\begin{array}{l}\text { Hypertonic } \\
\text { lactate saline }\end{array}$ & $R L$ & $\begin{array}{l}\text { Fluid volume } \\
\text { administered }\end{array}$ & Surrogate & 3 \\
\hline $\begin{array}{l}\text { Aboelatta } \\
\text { et al. [66] }\end{array}$ & 2013 & ISWCC & 2 & 30 & TBSA 25-60\% & $\begin{array}{l}\text { Fluid } \\
\text { resuscitation } \\
\text { guided by } \\
\text { PICCO }\end{array}$ & $\begin{array}{l}\text { Parkland } \\
\text { formula }\end{array}$ & $\begin{array}{l}\text { Fluid volume } \\
\text { administered }\end{array}$ & Surrogate & 3 \\
\hline $\begin{array}{l}\text { Arlati et al. } \\
{[67]}\end{array}$ & 2006 & ISWCC & 1 & 24 & TBSA > 20\% & $\begin{array}{l}\text { Permissive } \\
\text { hypovolaemia }\end{array}$ & $\begin{array}{l}\text { Parkland } \\
\text { formula }\end{array}$ & MODS & Surrogate & NA \\
\hline $\begin{array}{l}\text { Berger } \\
\text { et al. [68] }\end{array}$ & 2000 & ISWCC & 1 & 40 & TBSA > 25\% & $\begin{array}{l}\text { Bicarbonated } \\
0.9 \% \text { saline } \\
\text { (340 mmol) } \\
\text { solution }\end{array}$ & $R L$ & Mortality & $\begin{array}{l}\text { Patient- } \\
\text { centred }\end{array}$ & 10 \\
\hline $\begin{array}{l}\text { Gille et al. } \\
\text { [69] }\end{array}$ & 2014 & ISWCC & 1 & 80 & TBSA > 20\% & RA & $R L$ & SOFA score & Surrogate & 60 \\
\hline $\begin{array}{l}\text { Salinas } \\
\text { et al. [70] }\end{array}$ & 2011 & ISWCC & 1 & 70 & TBSA > 20\% & $\begin{array}{l}\text { Computer led } \\
\text { algorithm }\end{array}$ & $\begin{array}{l}\text { Parkland } \\
\text { formula }\end{array}$ & $\begin{array}{l}\text { Total crystalloid } \\
\text { volume in first } \\
48 \mathrm{~h}\end{array}$ & Surrogate & NA \\
\hline
\end{tabular}

$R C T$ randomised controlled trial, Pseudo RCT pseudo-randomised controlled trial, CSWCC clinical study with concurrent control, ISWCC intervention study without concurrent control, TBSA total burn surface area, RL Ringer's lactate solution, RA Ringer's acetate solution, FFP fresh frozen plasma, NS $0.9 \%$ sodium chloride solution, MODS multiple organ dysfunction score, IAP intra-abdominal pressure, SOFA sequential organ failure assessment, LFU longest follow-up

Table 4 Haemodynamic monitoring studies

\begin{tabular}{|c|c|c|c|c|c|c|c|c|c|c|}
\hline Author & Year & $\begin{array}{l}\text { Study } \\
\text { type }\end{array}$ & Centre(s) & Number & Population & Intervention & Control & $\begin{array}{l}\text { Primary } \\
\text { outcome }\end{array}$ & $\begin{array}{l}\text { Surrogate vs } \\
\text { patient- } \\
\text { centred }\end{array}$ & $\begin{array}{l}\text { LFU } \\
\text { (days) }\end{array}$ \\
\hline $\begin{array}{l}\text { Csontos } \\
\text { et al. } \\
{[71]}\end{array}$ & 2008 & $\mathrm{RCT}$ & 1 & 24 & TBSA > 15\% & $\mathrm{PICCO}$ & Urine output & $\begin{array}{l}\text { Central venous } \\
\text { O2 saturations }\end{array}$ & Surrogate & 3 \\
\hline $\begin{array}{l}\text { Holm } \\
\text { et al [72] }\end{array}$ & 2004 & $\mathrm{RCT}$ & 1 & 50 & TBSA $>20 \%$ & $\begin{array}{l}\text { Transpulmonary } \\
\text { thermodilution method } \\
\text { for CO }\end{array}$ & $\begin{array}{l}\text { Baxter formula } \\
\text { and urine } \\
\text { output }\end{array}$ & $\begin{array}{l}\text { In-hospital } \\
\text { mortality }\end{array}$ & Patient-centred & $>25$ \\
\hline $\begin{array}{l}\text { Tokarik } \\
\text { et al. } \\
\text { [73] }\end{array}$ & 2013 & $\mathrm{RCT}$ & 1 & 21 & $\begin{array}{l}\text { TBSA } 10-75 \% \\
\text { with burn } \\
\text { shock }\end{array}$ & LiDCO & $\begin{array}{l}\text { Physician led } \\
\text { resuscitation }\end{array}$ & $\begin{array}{l}\text { Cumulative } \\
\text { fluid balance }\end{array}$ & Surrogate & 37 \\
\hline $\begin{array}{l}\text { Holm } \\
\text { et al [74] }\end{array}$ & 2001 & $\begin{array}{l}\text { CSWC } \\
\text { C }\end{array}$ & 1 & 23 & $A B S I \geq 6$ & $\begin{array}{l}\text { Transpulmonary } \\
\text { thermodilution for } \mathrm{CO}\end{array}$ & $\begin{array}{l}\text { Pulmonary } \\
\text { artery catheter } \\
\text { for CO }\end{array}$ & Cardiac output & Surrogate & 3 \\
\hline
\end{tabular}


Table 5 Nutrition studies

\begin{tabular}{|c|c|c|c|c|c|c|c|c|c|c|}
\hline Author & Year & $\begin{array}{l}\text { Study } \\
\text { type }\end{array}$ & Centre(s) & Number & Population & Intervention & Control & $\begin{array}{l}\text { Primary } \\
\text { outcome }\end{array}$ & $\begin{array}{l}\text { Surrogate } \\
\text { vs } \\
\text { patient- } \\
\text { centred }\end{array}$ & $\begin{array}{l}\text { LFU } \\
\text { (days) }\end{array}$ \\
\hline $\begin{array}{l}\text { Berger et al. } \\
{[75]}\end{array}$ & 2007 & $\mathrm{RCT}$ & 1 & 21 & TBSA $>20 \%$ & $\begin{array}{l}\text { Intravenous trace } \\
\text { elements }\end{array}$ & Placebo & $\begin{array}{l}\text { Plasma/tissue } \\
\text { trace element } \\
\text { levels }\end{array}$ & Surrogate & 28 \\
\hline $\begin{array}{l}\text { Chen et al. } \\
{[76]}\end{array}$ & 2007 & $\mathrm{RCT}$ & 1 & 19 & TBSA $>30 \%$ & TPN & EN & Plasma motilin & Surrogate & 1 \\
\hline $\begin{array}{l}\text { Chuntrasakul } \\
\text { et al. [77] }\end{array}$ & 2003 & $\mathrm{RCT}$ & 1 & 36 & $\begin{array}{l}\text { TBSA }>30 \%[20] \\
\text { and non-burns } \\
\text { trauma patients } \\
{[16]}\end{array}$ & Immuno-EN & $\begin{array}{l}\text { Hypercaloric } \\
\text { EN }\end{array}$ & $\begin{array}{l}\text { Gastrointestinal } \\
\text { tolerance }\end{array}$ & $\begin{array}{l}\text { Patient- } \\
\text { centred }\end{array}$ & 4 \\
\hline $\begin{array}{l}\text { Garcia de } \\
\text { Lorenzo et al. } \\
{[78]}\end{array}$ & 2005 & $\mathrm{RCT}$ & 1 & 22 & $A B S I>7$ & High olive oil TPN & $\begin{array}{l}\text { Standard } \\
\text { TPN }\end{array}$ & TPN intake & Surrogate & 28 \\
\hline $\begin{array}{l}\text { Garrel et al. } \\
\text { [79] }\end{array}$ & 1995 & $\mathrm{RCT}$ & 1 & 43 & TBSA $>20 \%$ & $\begin{array}{l}\text { Low-fat diet with } \\
\text { or without fish oil }\end{array}$ & Standard EN & $\begin{array}{l}\text { Urine nitrogen } \\
\text { balance }\end{array}$ & Surrogate & 7 \\
\hline $\begin{array}{l}\text { Gottschlich } \\
\text { et al. [80] }\end{array}$ & 1990 & $\mathrm{RCT}$ & 1 & 50 & $\mathrm{TBSA}>10 \%$ & $\begin{array}{l}\text { High protein, low } \\
\text { linoleic acid EN }\end{array}$ & Standard EN & $\begin{array}{l}\text { Urine nitrogen } \\
\text { balance }\end{array}$ & Surrogate & 3 \\
\hline $\begin{array}{l}\text { Herndon et al. } \\
\text { [81] }\end{array}$ & 1989 & $\mathrm{RCT}$ & 1 & 39 & $\mathrm{TBSA}>50 \%$ & $E N+T P N$ & EN & Caloric intake & Surrogate & 3 \\
\hline $\begin{array}{l}\text { Herndon et al. } \\
\text { [82] }\end{array}$ & 1987 & $\mathrm{RCT}$ & 1 & 28 & TBSA $>50 \%$ & $\mathrm{EN}+\mathrm{TPN}$ & EN & $\begin{array}{l}\text { Monocyte } \\
\text { function }\end{array}$ & Surrogate & 2 \\
\hline $\begin{array}{l}\text { Larsson et al. } \\
\text { [83] }\end{array}$ & 1990 & $\mathrm{RCT}$ & 1 & 39 & TBSA $>30 \%$ & IV nitrogen + TPN & $\begin{array}{l}\text { Standard } \\
\text { TPN }\end{array}$ & Nitrogen balance & Surrogate & 46 \\
\hline $\begin{array}{l}\text { Ostadrahimi } \\
\text { et al. [84] }\end{array}$ & 2016 & $\mathrm{RCT}$ & 1 & 30 & TBSA > 20\% & EN & Normal diet & SOFA score & Surrogate & 2 \\
\hline $\begin{array}{l}\text { Peng et al. } \\
{[85]}\end{array}$ & 2004 & $\mathrm{RCT}$ & 1 & 48 & TBSA > 30\% & $\begin{array}{l}\text { EN + glutamine } \\
\text { supplementation }\end{array}$ & Standard EN & $\begin{array}{l}\text { Intestinal } \\
\text { permeability }\end{array}$ & Surrogate & $<1$ \\
\hline $\begin{array}{l}\text { Saffle et al. } \\
\text { [86] }\end{array}$ & 1997 & $\mathrm{RCT}$ & 1 & 49 & $\begin{array}{l}\text { Adult AND } \\
\text { paediatric TBSA } \\
0-20 \%, 21-40 \% \\
\text { and }>40 \%\end{array}$ & $\begin{array}{l}\text { Immunoenhancing } \\
\text { EN }\end{array}$ & Standard EN & Hospital LOS & $\begin{array}{l}\text { Patient- } \\
\text { centred }\end{array}$ & 3 \\
\hline $\begin{array}{l}\text { Tihista et al. } \\
\text { [87] }\end{array}$ & 2017 & $\mathrm{RCT}$ & 1 & 92 & TBSA > 15\% & Low-fat EN & Standard EN & $\begin{array}{l}\text { Infectious } \\
\text { complications }\end{array}$ & $\begin{array}{l}\text { Patient- } \\
\text { centred }\end{array}$ & NA \\
\hline Vicic et al. [88] & 2013 & $\mathrm{RCT}$ & 1 & 101 & TBSA $>20 \%$ & Early EN & Normal diet & Not specified & NA & 10 \\
\hline Yan et al. [89] & 2007 & $\mathrm{RCT}$ & 1 & 47 & TBSA $>50 \%$ & $\begin{array}{l}\text { L-arginine } \\
\text { supplementation to } \\
\text { EN }\end{array}$ & Standard EN & $\begin{array}{l}\text { Serum nitric } \\
\text { oxide level }\end{array}$ & Surrogate & 4 \\
\hline $\begin{array}{l}\text { Abribat et al. } \\
\text { [90] }\end{array}$ & 2000 & $\begin{array}{l}\text { Pseudo } \\
\text { RCT }\end{array}$ & 1 & 23 & TBSA $>25 \%$ & $\begin{array}{l}\text { Low-fat diet with } \\
\text { and without } \\
\text { addition of omega- } \\
3 \text { fatty acid }\end{array}$ & $\begin{array}{l}\text { Normal } \\
\text { enteral diet }\end{array}$ & $\begin{array}{l}\text { Insulin growth } \\
\text { factor } 1\end{array}$ & Surrogate & 28 \\
\hline Lam et al. [91] & 2008 & $\begin{array}{l}\text { Pseudo } \\
\text { RCT }\end{array}$ & 1 & 82 & TBSA 40-70\% & NG EN & TPN & $\begin{array}{l}\text { Plasma } \\
\text { immunoglobulins }\end{array}$ & Surrogate & 7 \\
\hline Peck et al. [92] & 2004 & $\begin{array}{l}\text { Pseudo } \\
\text { RCT }\end{array}$ & 1 & 27 & TBSA $>20 \%$ & Early EN & $\begin{array}{l}\text { Normal diet } \\
+ \text { EN if } \\
\text { required }\end{array}$ & REE & Surrogate & $>40$ \\
\hline $\begin{array}{l}\text { Peng et al. } \\
\text { [93] }\end{array}$ & 2001 & $\begin{array}{l}\text { Pseudo } \\
\text { RCT }\end{array}$ & 1 & 22 & $\mathrm{TBSA}>50 \%$ & Early EN & Delayed EN & $\begin{array}{l}\text { Intestinal } \\
\text { permeability }\end{array}$ & Surrogate & 5 \\
\hline $\begin{array}{l}\text { Saffle et al. } \\
\text { [94] }\end{array}$ & 1990 & $\begin{array}{l}\text { Pseudo } \\
\text { RCT }\end{array}$ & 1 & 45 & $\mathrm{TBSA}>25 \%$ & EN per REE & $\begin{array}{l}\text { EN per } \\
\text { Curreri } \\
\text { formula }\end{array}$ & Nitrogen balance & Surrogate & 1 \\
\hline $\begin{array}{l}\text { Wibbenmeyer } \\
\text { et al. [95] }\end{array}$ & 2006 & $\begin{array}{l}\text { Pseudo } \\
\text { RCT }\end{array}$ & 1 & 23 & $\mathrm{TBSA}>20 \%$ & $\begin{array}{l}\mathrm{EN}+\text { fish oil and } \\
\text { arginine }\end{array}$ & Standard EN & $\begin{array}{l}\text { Time to healing } \\
\text { first donor graft } \\
\text { site }\end{array}$ & $\begin{array}{l}\text { Patient- } \\
\text { centred }\end{array}$ & 3 \\
\hline Zhou et al. & 2003 & Pseudo & 1 & 41 & $\mathrm{TBSA}>50 \%$ & $\mathrm{EN}+$ glutamine & Standard EN & Plasma amino & Surrogate & 30 \\
\hline
\end{tabular}


Table 5 Nutrition studies (Continued)

\begin{tabular}{|c|c|c|c|c|c|c|c|c|c|c|}
\hline Author & Year & $\begin{array}{l}\text { Study } \\
\text { type }\end{array}$ & Centre(s) & Number & Population & Intervention & Control & $\begin{array}{l}\text { Primary } \\
\text { outcome }\end{array}$ & $\begin{array}{l}\text { Surrogate } \\
\text { vs } \\
\text { patient- } \\
\text { centred }\end{array}$ & $\begin{array}{l}\text { LFU } \\
\text { (days) }\end{array}$ \\
\hline [96] & & RCT & & & & & & acid levels & & \\
\hline $\begin{array}{l}\text { Brown et al. } \\
{[97]}\end{array}$ & 1990 & CSWCC & 1 & 20 & TBSA $>10 \%$ & $\begin{array}{l}\text { TPN + modified } \\
\text { amino acids }\end{array}$ & $\begin{array}{l}\text { Standard } \\
\text { TPN }\end{array}$ & Nitrogen balance & Surrogate & 28 \\
\hline $\begin{array}{l}\text { Dhanraj et al. } \\
\text { [98] }\end{array}$ & 1997 & CSWCC & 1 & 20 & TBSA $20-50 \%$ & $\begin{array}{l}\text { Hospital-prepared } \\
\text { high-energy diet }\end{array}$ & $\begin{array}{l}\text { Commercial } \\
\text { EN }\end{array}$ & $\begin{array}{l}\text { Weight gain } \\
\text { (percent change) }\end{array}$ & Surrogate & $>28$ \\
\hline $\begin{array}{l}\text { Falder et al. } \\
\text { [99] }\end{array}$ & 2010 & CSWCC & 1 & 20 & TBSA $>15 \%$ & $\mathrm{EN}+$ thiamine & $\begin{array}{l}\text { Normal EN } \\
\text { or TPN }\end{array}$ & $\begin{array}{l}\text { Serum thiamine } \\
\text { level }\end{array}$ & Surrogate & 28 \\
\hline $\begin{array}{l}\text { Hiebert et al. } \\
\text { [100] }\end{array}$ & 1980 & CSWCC & 1 & 76 & TBSA $>10 \%$ & $\begin{array}{l}\text { Intermittent bolus } \\
\text { NG feeds }\end{array}$ & $\begin{array}{l}\text { Continuous } \\
\text { NG feeds }\end{array}$ & Stool frequency & $\begin{array}{l}\text { Patient- } \\
\text { centred }\end{array}$ & NA \\
\hline $\begin{array}{l}\text { Shields et al. } \\
\text { [101] }\end{array}$ & 2014 & CSWCC & 1 & 14 & TBSA $>35 \%$ & $\begin{array}{l}\text { Re-initiation of EN } \\
\text { at goal rate }\end{array}$ & $\begin{array}{l}\text { Slow re- } \\
\text { initiation of } \\
\text { EN }\end{array}$ & $\begin{array}{l}\text { Time to reach } \\
\text { goal rate }\end{array}$ & Surrogate & $>60$ \\
\hline $\begin{array}{l}\text { Gudaviciene } \\
\text { et al. [102] }\end{array}$ & 2004 & ISWCC & 1 & 138 & TBSA $>10 \%$ & EN + normal diet & $\begin{array}{l}\text { Nil feed } \\
\text { during acute } \\
\text { phase }\end{array}$ & $\begin{array}{l}\text { Incidence } \\
\text { pneumonia }\end{array}$ & $\begin{array}{l}\text { Patient- } \\
\text { centred }\end{array}$ & NA \\
\hline $\begin{array}{l}\text { Kesey et al. } \\
{[103]}\end{array}$ & 2013 & ISWCC & 1 & 76 & TBSA $>25 \%$ & Early EN & $\begin{array}{l}\text { Standard EN } \\
\text { feed } \\
\text { protocol }\end{array}$ & $\begin{array}{l}\text { Time to initiation } \\
\text { of feeding }\end{array}$ & Surrogate & 7 \\
\hline $\begin{array}{l}\text { Soguel et al. } \\
{[104]}\end{array}$ & 2008 & ISWCC & 1 & 40 & TBSA $>20 \%$ & $\begin{array}{l}\text { Glutamine } \\
\text { supplementation to } \\
\text { EN }\end{array}$ & Standard EN & SOFA score & Surrogate & 5 \\
\hline $\begin{array}{l}\text { Varon et al. } \\
{[105]}\end{array}$ & 2017 & ISWCC & 1 & 33 & TBSA $>20 \%$ & $\begin{array}{l}\text { Continuous EN } \\
\text { feeds }\end{array}$ & $\begin{array}{l}\text { Fasted } \\
\text { during } \\
\text { surgery }\end{array}$ & $\begin{array}{l}\text { Nutritional } \\
\text { targets }\end{array}$ & Surrogate & 36 \\
\hline
\end{tabular}

$R C T$ randomised controlled trial, Pseudo $R C T$ pseudo-randomised controlled trial, CSWCC clinical study with concurrent control, ISWCC intervention study without concurrent control, TBSA total burn surface area, EN enteral nutrition, TPN total parenteral nutrition, REE resting energy expenditure, NG nasogastric , LFU longest follow-up

Table 6 Surgical timing studies

\begin{tabular}{|c|c|c|c|c|c|c|c|c|c|c|}
\hline Author & Year & $\begin{array}{l}\text { Study } \\
\text { type }\end{array}$ & Centre(s) & Number & Population & Intervention & Control & $\begin{array}{l}\text { Primary } \\
\text { outcome }\end{array}$ & $\begin{array}{l}\text { Surrogate } \\
\text { vs patient- } \\
\text { centred }\end{array}$ & $\begin{array}{l}\text { LFU } \\
\text { (days) }\end{array}$ \\
\hline $\begin{array}{l}\text { Rutan et al. } \\
\text { [106] }\end{array}$ & 1986 & $\begin{array}{l}\text { Pseudo } \\
\text { RCT }\end{array}$ & 1 & 13 & TBSA > 50\% & Early E\&G & $\begin{array}{l}\text { Conservative } \\
\text { management }\end{array}$ & $\begin{array}{l}\text { Basal } \\
\text { metabolism }\end{array}$ & Surrogate & 30 \\
\hline $\begin{array}{l}\text { Sorensen } \\
\text { [107] }\end{array}$ & 1979 & $\begin{array}{l}\text { Pseudo } \\
\text { RCT }\end{array}$ & 1 & 108 & $\begin{array}{l}\text { Adult and } \\
\text { paediatric patients } \\
\text { mostly TBSA > 40\% }\end{array}$ & Early E\&G & $\begin{array}{l}\text { Surgery } 10-14 \\
\text { days post injury }\end{array}$ & Mortality & $\begin{array}{l}\text { Patient- } \\
\text { centred }\end{array}$ & NA \\
\hline $\begin{array}{l}\text { Guo et al. } \\
\text { [108] }\end{array}$ & 1995 & CSWCC & 1 & 50 & TBSA > 20\% & Early E\&G & $\begin{array}{l}\text { Standard surgical } \\
\text { timing ( } 4 \text { days } \\
\text { post burn) }\end{array}$ & $\begin{array}{l}\text { Haemodynamic } \\
\text { parameters }\end{array}$ & Surrogate & $>40$ \\
\hline $\begin{array}{l}\text { Kisslaogglu } \\
\text { et al. [109] }\end{array}$ & 1997 & CSWCC & 1 & 54 & $\begin{array}{l}\text { Adult and } \\
\text { paediatric TBSA 40- } \\
80 \%\end{array}$ & Early E\&G & $\begin{array}{l}\text { Late surgery or } \\
\text { conservative } \\
\text { management }\end{array}$ & Mortality & $\begin{array}{l}\text { Patient- } \\
\text { centred }\end{array}$ & $\begin{array}{l}180 \\
\text { days (6 } \\
\text { months) }\end{array}$ \\
\hline $\begin{array}{l}\text { Puri et al. } \\
{[110]}\end{array}$ & 2016 & CSWCC & 1 & 20 & TBSA > 20\% & Early E\&G & $\begin{array}{l}\text { Conservative } \\
\text { management }\end{array}$ & Blood loss & Surrogate & 42 \\
\hline
\end{tabular}

Pseudo RCT pseudo-randomised controlled trial, CSWCC clinical study with concurrent control, TBSA total burn surface area, E\&G excision and grafting, LFU longest follow-up 
Table 7 Transfusion studies

\begin{tabular}{|c|c|c|c|c|c|c|c|c|c|c|}
\hline Author & Year & $\begin{array}{l}\text { Study } \\
\text { type }\end{array}$ & Centre(s) & Number & Population & Intervention & Control & $\begin{array}{l}\text { Primary } \\
\text { outcome }\end{array}$ & $\begin{array}{l}\text { Surrogate } \\
\text { vs patient- } \\
\text { centred }\end{array}$ & $\begin{array}{l}\text { LFU } \\
\text { (days) }\end{array}$ \\
\hline $\begin{array}{l}\text { Johannson } \\
\text { et al. [111] }\end{array}$ & 2007 & $\mathrm{RCT}$ & 1 & 18 & TBSA $>10 \%$ & $\begin{array}{l}\text { Recombinant factor } \\
\text { VIla during burn } \\
\text { E\&G }\end{array}$ & Placebo & $\begin{array}{l}\text { Transfusion } \\
\text { requirement }\end{array}$ & Surrogate & 30 \\
\hline $\begin{array}{l}\text { Mzezewa } \\
\text { et al. [112] }\end{array}$ & 2004 & $\mathrm{RCT}$ & 1 & 51 & $\begin{array}{l}\text { Adult AND } \\
\text { paediatric (mostly } \\
\text { adult) TBSA > 10\% }\end{array}$ & Pre-op terlipressin & Placebo & Blood loss & Surrogate & NA \\
\hline $\begin{array}{l}\text { Palmieri } \\
\text { et al. [113] }\end{array}$ & 2017 & $\mathrm{RCT}$ & 18 & 347 & TBSA $>20 \%$ & $\begin{array}{l}\text { Restrictive } \\
\text { transfusion strategy } \\
\text { (Hb target 70-80 g/ } \\
\text { L) }\end{array}$ & $\begin{array}{l}\text { Liberal transfusion } \\
\text { strategy }(\mathrm{Hb} \text { target } \\
100-110 \mathrm{~g} / \mathrm{L})\end{array}$ & $\begin{array}{l}\text { Number of } \\
\text { blood stream } \\
\text { infections }\end{array}$ & $\begin{array}{l}\text { Patient- } \\
\text { centred }\end{array}$ & 31 \\
\hline $\begin{array}{l}\text { Schaden } \\
\text { et al. [114] }\end{array}$ & 2012 & $\mathrm{RCT}$ & 1 & 30 & $\mathrm{TBSA}>25 \%$ & $\begin{array}{l}\text { ROTEM-guided } \\
\text { algorithm }\end{array}$ & $\begin{array}{l}\text { Standard } \\
\text { transfusion strategy }\end{array}$ & $\begin{array}{l}\text { Transfusion } \\
\text { requirements }\end{array}$ & Surrogate & 3 \\
\hline $\begin{array}{l}\text { Still et al. } \\
\text { [115] }\end{array}$ & 1995 & $\mathrm{RCT}$ & 7 & 40 & TBSA 25-65\% & rh-EPO & Standard care & $\begin{array}{l}\mathrm{Hb} \text { pre and } \\
\text { post op }\end{array}$ & Surrogate & 30 \\
\hline $\begin{array}{l}\text { Lundy } \\
\text { et al. [116] }\end{array}$ & 2010 & $\begin{array}{l}\text { CSWC } \\
\text { C }\end{array}$ & 1 & 104 & TBSA $>30 \%$ & rh-EPO & Standard care & $\begin{array}{l}\mathrm{Hb} \text { pre and } \\
\text { post op }\end{array}$ & Surrogate & $>60$ \\
\hline $\begin{array}{l}\text { Imai et al. } \\
{[117]}\end{array}$ & 2007 & ISWCC & 1 & 14 & TBSA $<30 \%$ & $\begin{array}{l}\text { Autologous PRC } \\
\text { transfusion }\end{array}$ & $\begin{array}{l}\text { Allogeneic PRC } \\
\text { transfusion }\end{array}$ & Haematocrit & Surrogate & 14 \\
\hline $\begin{array}{l}\text { Kowal-vern } \\
\text { et al. [118] }\end{array}$ & 2000 & ISWCC & 1 & 18 & TBSA $>20 \%$ & ATIII infusion & Standard care & ATIII levels & Surrogate & 20 \\
\hline
\end{tabular}

$R C T$ randomised controlled trial, CSWCC clinical study with concurrent control, ISWCC intervention study without concurrent control, TBSA total burn surface area, E\&G excision and grafting, ROTEM rotational thromboelastometry, rh-EPO recombinant human erythropoietin, PRC packed red cells, ATIII antithrombin III, LFU longest follow-up

Table 8 Ventilation studies

\begin{tabular}{|c|c|c|c|c|c|c|c|c|c|c|}
\hline Author & Year & $\begin{array}{l}\text { Study } \\
\text { type }\end{array}$ & Centre(s) & Number & Population & Intervention & Control & $\begin{array}{l}\text { Primary } \\
\text { outcome }\end{array}$ & $\begin{array}{l}\text { Surrogate } \\
\text { vs } \\
\text { patient- } \\
\text { centred }\end{array}$ & $\begin{array}{l}\text { LFU } \\
\text { (days) }\end{array}$ \\
\hline $\begin{array}{l}\text { Elsharnouby } \\
\text { et al. [119] }\end{array}$ & 2014 & $\mathrm{RCT}$ & 1 & 29 & TBSA > 15\% & $\begin{array}{l}\text { Nebulised heparin } \\
\text { sulphate } 10,000 \text { IU } \\
\text { with NAC }\end{array}$ & $\begin{array}{l}\text { Nebulised } \\
\text { heparin sulphate } \\
5000 \text { IU with } \\
\text { NAC }\end{array}$ & $\begin{array}{l}\text { Lung injury } \\
\text { score }\end{array}$ & Surrogate & 35 \\
\hline $\begin{array}{l}\text { Reper et al. } \\
{[120]}\end{array}$ & 2002 & $\mathrm{RCT}$ & 1 & 35 & TBSA $>20 \%$ & HFPV & $\begin{array}{l}\text { Conventional } \\
\text { mechanical } \\
\text { ventilation }\end{array}$ & $\mathrm{FiO} 2$ & Surrogate & 5 \\
\hline $\begin{array}{l}\text { Chung et al. } \\
{[121]}\end{array}$ & 2010 & $\begin{array}{l}\text { Pseudo } \\
\text { RCT }\end{array}$ & 1 & 62 & TBSA $>30 \%$ & HFPV & $\begin{array}{l}\text { Low tidal } \\
\text { volume } \\
\text { ventilation }\end{array}$ & $\begin{array}{l}\text { Ventilator- } \\
\text { free days in } \\
\text { first } 28 \text { days }\end{array}$ & $\begin{array}{l}\text { Patient- } \\
\text { centred }\end{array}$ & 28 \\
\hline $\begin{array}{l}\text { Mcginn } \\
\text { et al. [122] }\end{array}$ & 2019 & CSWCC & 1 & 48 & $\begin{array}{l}\text { Mechanically } \\
\text { ventilated with } \\
\text { inhalational } \\
\text { injury }\end{array}$ & $\begin{array}{l}\text { Nebulised heparin } \pm \\
\text { NAC and albuterol }\end{array}$ & $\begin{array}{l}\text { Albuterol } \pm \\
\text { ipratropium }\end{array}$ & $\begin{array}{l}\text { Duration of } \\
\text { mechanical } \\
\text { ventilation }\end{array}$ & $\begin{array}{l}\text { Patient- } \\
\text { centred }\end{array}$ & NA \\
\hline $\begin{array}{l}\text { Miller et al. } \\
\text { [123] }\end{array}$ & 2009 & ISWCC & 1 & 30 & $\begin{array}{l}\text { Inhalational burn } \\
\text { injury }\end{array}$ & $\begin{array}{l}\text { Nebulised heparin } \\
\text { sulphate 10,000 IU } \\
\text { with NAC and } \\
\text { albuterol }\end{array}$ & $\begin{array}{l}\text { Nebulised } \\
\text { albuterol }\end{array}$ & $\begin{array}{l}\text { Lung injury } \\
\text { score }\end{array}$ & Surrogate & 7 \\
\hline
\end{tabular}


Table 9 Numbers and percentages of papers with OMERACT outcome reporting

\begin{tabular}{lllll}
\hline \multirow{2}{*}{ Study domain, (total no.) } & \multicolumn{2}{l}{ OMERACT outcomes } \\
\cline { 2 - 5 } Death (\%) & Life impact (\%) & Resource/economic (\%) & Pathophysiological manifestations (\%) \\
\hline Analgesia [19] & $1(5)$ & $19(100)$ & $2(11)$ & $19(100)$ \\
Fluid resuscitation [26] & $15(57)$ & $0(0)$ & $4(15)$ & $25(96)$ \\
Haemodynamic monitoring [4] & $3(75)$ & $0(0)$ & $2(50)$ & $4(100)$ \\
Nutrition [31] & $21(67)$ & $3(10)$ & $14(45)$ & $30(97)$ \\
Surgical timing [5] & $3(60)$ & $2(40)$ & $2(40)$ & $3(60)$ \\
Transfusion strategies [8] & $4(50)$ & $2(25)$ & $4(50)$ & $7(88)$ \\
Ventilation strategies [5] & $5(100)$ & $1(20)$ & $1(20)$ & $5(100)$ \\
Total [98] & $52(53)$ & $27(28)$ & $29(30)$ & $93(95)$ \\
\hline
\end{tabular}

Table 10 Numbers and percentages of papers with Falder outcome reporting

\begin{tabular}{|c|c|c|c|c|c|c|c|}
\hline \multirow[b]{2}{*}{ Study domain (total no.) } & \multicolumn{7}{|c|}{ Falder outcomes } \\
\hline & $\begin{array}{l}\text { Skin } \\
(n)\end{array}$ & $\begin{array}{l}\text { NM function } \\
(n)\end{array}$ & $\begin{array}{l}\text { Sensory/pain } \\
(n)\end{array}$ & $\begin{array}{l}\text { Psychological } \\
\text { (n) }\end{array}$ & $\begin{array}{l}\text { Physical function } \\
\text { (n) }\end{array}$ & $\begin{array}{l}\text { Community } \\
(n)\end{array}$ & $\begin{array}{l}\text { Quality of life } \\
(n)\end{array}$ \\
\hline Analgesia [19] & 0 & 0 & 19 & 2 & 0 & 0 & 1 \\
\hline Fluid resuscitation [26] & 0 & 0 & 0 & 0 & 0 & 0 & 0 \\
\hline $\begin{array}{l}\text { Haemodynamic monitoring } \\
{[4]}\end{array}$ & 0 & 0 & 0 & 0 & 0 & 0 & 0 \\
\hline Nutrition [31] & 3 & 0 & 0 & 0 & 0 & 0 & 0 \\
\hline Surgical timing [5] & 2 & 0 & 0 & 0 & 1 & 0 & 0 \\
\hline Transfusion strategies [8] & 2 & 0 & 0 & 0 & 0 & 0 & 0 \\
\hline Ventilation strategies [5] & 0 & 0 & 0 & 0 & 0 & 0 & 0 \\
\hline Total [98] & $7(\%)$ & $0(0 \%)$ & 19 (19\%) & $2(2 \%)$ & $1(1 \%)$ & $0(0 \%)$ & $1(1 \%)$ \\
\hline
\end{tabular}




\section{Appendix B}

\section{Search strategy MeSH terms}

\section{PubMed}

((()((()isotonic solution OR crystalloid OR saline OR intravenous fluid)) OR (analgesia OR anaesthesia and analgesia OR pain management)) OR physiologic monitoring) OR (pulmonary ventilation OR invasive ventilation OR non-invasive ventilation)) OR (blood transfusion OR blood product transfusion OR transfusion)) OR enteral nutrition) AND (burns OR thermal injury OR burns injury OR chemical injury OR electrical injury)) AND (intensive care OR critical care OR intensive care unit OR critically ill OR critical illness)

\section{Medline}

((()((isotonic solution OR crystalloid OR intravenous fluid OR saline) OR (analgesia OR anaesthesia OR pain management)) OR (mechanical ventilation OR pulmonary ventilation OR artificial respiration))) OR (blood transfusion OR blood product transfusion OR packed cell transfusion OR transfusion)))) OR (enteral nutrition OR nutrition supplement OR parenteral nutrition))))) OR (surgery OR debridement OR timing of surgery OR skin graft OR cosmetic surgery)))))) AND (burns OR chemical burn OR thermal injury OR heat injury OR chemical injury) AND (intensive care OR critical care OR critical illness OR intensive care unit or ICU)

\section{Appendix C}

Pre-specified protocol: Patient-centred core outcomes are under-reported in the critical care burns literature: a systematic review

\section{Aim:}

To establish what outcomes are reported in published research that pertains to the intensive care management of patients with severe burns.

The management areas are:

1. Fluid resuscitation in the acute burn phase

2. Analgesia

3. Haemodynamic monitoring and end points to target

4. Ventilation strategies

5. Transfusion targets

6. Enteral nutrition composition and targets

7. Surgery-debridement and/or grafting

\section{Methods:}

\section{Step 1:}

Three independent investigators carry out detailed search of literature for suitable articles. The search strategies used are outlined in Appendix A.
Also, search bibliographies of recent review articles are on the topic of acute burns management for additional articles not already found.

\section{Step 2:}

Refine search results to only include studies that fit strict inclusion criteria:

- Adult

- Human

- The subject group are patients with thermal burns and/or inhalational injury admitted to Intensive Care units.

- Level II or III evidence (NHMRC ${ }^{1}$ and OCEBM ${ }^{2}$ ) which includes randomised controlled trials and cohort/comparative studies

- Related to one or more of the seven management areas (see above)

- Time frame January 11960 to December 312019

\section{Step 3:}

Information to be collected from each article is:

- Author

- Year published

- Study type

- Patient population

- Which intensive care management area

- Primary outcomes and whether surrogate or patientcentred

- Timing of longest follow-up

\section{Step 4:}

Classify the outcomes reported according to the OMERACT $^{3}$ and Falder ${ }^{4}$ Frameworks.

The OMERACT framework classifies the outcomes reported into four domains which are:

- Death

- Life impact

- Resource use/economic impact

- Pathophysiological manifestations

${ }^{1}$ National Health and Medical Research Council ${ }^{2}$ Oxford Centre for Evidence-based Medicine ${ }^{3}$ Boers M, Kirwan JR, Wells G, Beaton D, Gossec L, d'Agostino MA, et al. Developing core outcome measurement sets for clinical trials: OMERACT filter 2.0. J Clin Epidemiol. 2014;67 [7]:745-53.

${ }^{4}$ Falder S, Browne A, Edgar D, Staples E, Fong J, Rea S, et al. Core outcomes for adult burn survivors: a clinical overview. Burns. 2009;35 [5]:618-41 
The burns specific outcome reporting framework proposed by Falder includes patient specific functional and psychosocial outcomes. These are:

- Skin

- Sensory and pain

- Psychological function

- Physical role function

- Community participation

- Perceived quality of life

\section{Step 5:}

Quantitative and qualitative data from the studies to be derived and tabulated with percentages and proportions reported.

\section{Acknowledgements}

Not applicable.

\section{Authors' contributions}

All authors contributed to study concept and design, data search and extraction and manuscript preparation and review.

\section{Funding}

This research did not receive any grant from funding agencies in the public, commercial or not-for-profit sectors.

\section{Availability of data and materials}

Data for preparation of this manuscript has been derived from the open access literature.

\section{Declarations}

Ethics approval and consent to participate

Not applicable.

\section{Consent for publication}

All authors give consent for publication of this manuscript.

\section{Competing interests}

The authors declare no competing interests.

\section{Author details}

'Malcolm Fisher Department of Intensive Care, The Royal North Shore Hospital, St Leonards, NSW 2065, Australia. ${ }^{2}$ The University of New South Wales, Kensington, Sydney, NSW, Australia. ${ }^{3}$ Department of Intensive Care, Orange Base Hospital, Orange, NSW, Australia. ${ }^{4}$ Northern Clinical School, University of Sydney, Sydney, NSW, Australia. ${ }^{5}$ The George Institute for Global Health, Sydney, NSW, Australia.

Received: 23 March 2021 Accepted: 12 February 2022

Published online: 04 March 2022

\section{References}

1. Snell JA, Loh NH, Mahambrey T, Shokrollahi K. Clinical review: the critical care management of the burn patient. Crit Care. 2013;17(5):241.

2. Wood FM. Quality assurance in burn patient care: the James Laing Memorial Essay, 1994. Burns. 1995;21(8):563-8.

3. Brown T, Mills S, Muller M. If it can't be measured it can't be managed. The paucity of outcome measures in burn care. Burns. 2003;29(8):757.

4. Brown TL, Muller MJ, Mills SJ. What does the black cat look like. Burns. 2004; 30(4):303.

5. Clarke M. Standardising outcomes for clinical trials and systematic reviews. Trials. 2007;8:39.

6. Kirkham JJ, Boers M, Tugwell P, Clarke M, Williamson PR. Outcome measures in rheumatoid arthritis randomised trials over the last 50 years. Trials. 2013; 14:324.
7. Boers M, Kirwan JR, Wells G, Beaton D, Gossec L, d'Agostino MA, et al. Developing core outcome measurement sets for clinical trials: OMERACT filter 2.0. J Clin Epidemiol. 2014;67(7):745-53.

8. de Wit M, Cooper C, Reginster JY, Group W-EW. Practical guidance for patient-centred health research. Lancet. 2019;393(10176):1095-6.

9. Moza A, Benstoem C, Autschbach R, Stoppe C, Goetzenich A. A core outcome set for all types of cardiac surgery effectiveness trials: a study protocol for an international eDelphi survey to achieve consensus on what to measure and the subsequent selection of measurement instruments. Trials. 2015;16:545

10. Hall NJ, Kapadia MZ, Eaton S, Chan WW, Nickel C, Pierro A, et al. Outcome reporting in randomised controlled trials and meta-analyses of appendicitis treatments in children: a systematic review. Trials. 2015;16:275.

11. Blackwood B, Marshall J, Rose L. Progress on core outcome sets for critical care research. Curr Opin Crit Care. 2015;21(5):439-44.

12. Falder S, Browne A, Edgar D, Staples E, Fong J, Rea S, et al. Core outcomes for adult burn survivors: a clinical overview. Burns. 2009;35(5):618-41.

13. Shamseer L, Moher D, Clarke M, Ghersi D, Liberati A, Petticrew M, et al. Preferred reporting items for systematic review and meta-analysis protocols (PRISMA-P) 2015: elaboration and explanation. BMJ. 2015;350:g7647.

14. NHMRC additional levels of evidence and grades for recommendations for developers of guidelines.

15. Guyatt GH, Oxman AD, Kunz R, Atkins D, Brozek J, Vist G, et al. GRADE guidelines: 2. Framing the question and deciding on important outcomes. J Clin Epidemiol. 2011;64(4):395-400.

16. Møller MH. Patient-important outcomes and core outcome sets: increased attention needed! Br J Anaesth. 2019;122(4):408-10.

17. Holley AD, Reade MC, Lipman J, Delaney A, Udy A, Lee R, et al. Survey of critical care practice in Australian and New Zealand burn referral centres. Crit Care Resusc. 2019;21(4):303-4.

18. Angus DC, Carlet J, Participants BR. Surviving intensive care: a report from the 2002 Brussels Roundtable. Intensive Care Med. 2003:29(3):368-77.

19. Haywood K, Whitehead L, Nadkarni VM, Achana F, Beesems S, Böttiger BW, et al. COSCA (Core Outcome Set for Cardiac Arrest) in adults: an advisory statement from the International Liaison Committee on Resuscitation. Resuscitation. 2018:127:147-63.

20. Needham DM, Sepulveda KA, Dinglas VD, Chessare CM, Friedman LA, Bingham CO, et al. Core outcome measures for clinical research in acute respiratory failure survivors. An International Modified Delphi Consensus Study. Am J Respir Crit Care Med. 2017:196(9):1122-30.

21. Andersen CR, Fitzgerald E, Delaney A, Finfer $S$. A systematic review of outcome measures employed in aneurysmal subarachnoid hemorrhage (aSAH) clinical research. Neurocrit Care. 2019;30(3):534-41.

22. Connolly B, Denehy L, Hart N, Pattison N, Williamson P, Blackwood B. Physical Rehabilitation Core Outcomes In Critical illness (PRACTICE): protocol for development of a core outcome set. Trials. 2018;19(1):294.

23. Rose L, Agar M, Burry LD, Campbell N, Clarke M, Lee J, et al. Development of core outcome sets for effectiveness trials of interventions to prevent and/ or treat delirium (Del-COrS): study protocol. BMJ Open. 2017:7(9):e016371.

24. Cooney RM, Warren BF, Altman DG, Abreu MT, Travis SP. Outcome measurement in clinical trials for ulcerative colitis: towards standardisation. Trials. 2007;8:17.

25. Sinha IP, Gallagher R, Williamson PR, Smyth RL. Development of a core outcome set for clinical trials in childhood asthma: a survey of clinicians, parents, and young people. Trials. 2012;13:103.

26. Choiniere M, Grenier R, Paquette C. Patient-controlled analgesia: a doubleblind study in burn patients. Anaesthesia. 1992:47(6):467-72.

27. Cuignet O, Pirson J, Boughrouph J, Duville D. The efficacy of continuous fascia iliaca compartment block for pain management in burn patients undergoing skin grafting procedures. Anesth Analg. 2004;98(4):1077-81 table of contents.

28. Everett JJ, Patterson DR, Burns GL, Montgomery B, Heimbach D. Adjunctive interventions for burn pain control: comparison of hypnosis and ativan: the 1993 Clinical Research Award. J Burn Care Rehabil. 1993;14(6):676-83.

29. Finn J, Wright J, Fong J, Mackenzie E, Wood F, Leslie G, et al. A randomised crossover trial of patient controlled intranasal fentanyl and oral morphine for procedural wound care in adult patients with burns. Burns. 2004;30(3): 262-8.

30. Gray P, Kirby J, Smith MT, Cabot PJ, Williams B, Doecke J, et al. Pregabalin in severe burn injury pain: a double-blind, randomised placebo-controlled trial. Pain. 2011;152(6):1279-88. 
31. Gündüz M, Sakalli S, Güneş Y, Kesiktaş E, Ozcengiz D, Işik G. Comparison of effects of ketamine, ketamine-dexmedetomidine and ketamine-midazolam on dressing changes of burn patients. J Anaesthesiol Clin Pharmacol. 2011; 27(2):220-4.

32. Jellish WS, Gamelli RL, Furry PA, McGill VL, Fluder EM. Effect of topical local anesthetic application to skin harvest sites for pain management in burn patients undergoing skin-grafting procedures. Ann Surg. 1999; 229(1):115-20

33. Kundra P, Velayudhan S, Krishnamachari S, Gupta SL. Oral ketamine and dexmedetomidine in adults' burns wound dressing--a randomized double blind cross over study. Burns. 2013;39(6):1150-6.

34. Lee JJ, Marvin JA, Heimbach DM. Effectiveness of nalbuphine for relief of burn debridement pain. J Burn Care Rehabil. 1989;10(3):241-6.

35. Patterson DR, Ptacek JT, Carrougher GJ, Sharar SR. Lorazepam as an adjunct to opioid analgesics in the treatment of burn pain. Pain. 1997; 72(3):367-74.

36. Prakash S, Fatima T, Pawar M. Patient-controlled analgesia with fentanyl for burn dressing changes. Anesth Analg. 2004;99(2):552-5 table of contents.

37. Raza MS, Nazim T, Khan FA. Comparison of bupivacaine moistened dressing and conventional dressing for pain relief on skin graft donor sites. J Coll Physicians Surg Pak. 2014;24(6):416-9.

38. Wasiak J, Spinks A, Costello V, Ferraro F, Paul E, Konstantatos A, et al Adjuvant use of intravenous lidocaine for procedural burn pain relief: a randomized double-blind, placebo-controlled, cross-over trial. Burns. 2011; 37(6):951-7.

39. Wibbenmeyer L, Eid A, Liao J, Heard J, Horsfield A, Kral L, et al. Gabapentin is ineffective as an analgesic adjunct in the immediate postburn period. J Burn Care Res. 2014;35(2):136-42.

40. Yuxiang L, Lu T, Jianqiang Y, Xiuying D, Wanfang Z, Wannian Z, et al. Analgesia effect of a fixed nitrous oxide/oxygen mixture on burn dressing pain: study protocol for a randomized controlled trial. Trials. 2012;13:67.

41. Zor F, Ozturk S, Bilgin F, Isik S, Cosar A. Pain relief during dressing changes of major adult burns: ideal analgesic combination with ketamine. Burns. 2010;36(4):501-5.

42. Foertsch CE, O'Hara MW, Kealey GP, Foster LD, Schumacher EA. A quasiexperimental, dual-center study of morphine efficacy in patients with burns. J Burn Care Rehabil. 1995;16(2 Pt 1):118-26.

43. Nilsson A, Steinvall I, Bak Z, Sjoberg F. Patient controlled sedation using a standard protocol for dressing changes in burns: patients' preference, procedural details and a preliminary safety evaluation. Burns. 2008;34(7): 929-34.

44. Berger MM, Davadant M, Marin C, Wasserfallen JB, Pinget C, Maravic $P$, et al. Impact of a pain protocol including hypnosis in major burns. Burns. 2010; 36(5):639-46.

45. Bechir M, Puhan MA, Fasshauer M, Schuepbach RA, Stocker R, Neff TA. Early fluid resuscitation with hydroxyethyl starch 130/0.4 (6\%) in severe burn injury: a randomized, controlled, double-blind clinical trial. Crit Care. 2013; 17(6):R299.

46. Bedi MK, Sarabahi S, Agrawal K. New fluid therapy protocol in acute burn from a tertiary burn care centre. Burns. 2019;45(2):335-40.

47. Belba MK, Petrela EY, Belba GP. Comparison of hypertonic vs isotonic fluids during resuscitation of severely burned patients. Am J Emerg Med. 2009; 27(9):1091-6.

48. Bortolani A, Governa M, Barisoni D. Fluid replacement in burned patients. Acta Chir Plast. 1996;38(4):132-6.

49. Cooper AB, Cohn SM, Zhang HS, Hanna K, Stewart TE, Slutsky AS. Five percent albumin for adult burn shock resuscitation: lack of effect on daily multiple organ dysfunction score. Transfusion. 2006;46(1):80-9.

50. Goodwin CW, Dorethy J, Lam V, Pruitt BA Jr. Randomized trial of efficacy of crystalloid and colloid resuscitation on hemodynamic response and lung water following thermal injury. Ann Surg. 1983;197(5):520-31.

51. Gunn ML, Hansbrough JF, Davis JW, Furst SR, Field TO. Prospective, randomized trial of hypertonic sodium lactate versus lactated Ringer's solution for burn shock resuscitation. J Trauma. 1989;29(9):1261-7.

52. Hall KV, Sørensen B. The treatment of burn shock: results of a 5-year randomized, controlled clinical trial of Dextran 70 v. Ringer lactate solution. Burns J. 1978.

53. Huang Y, Yan B, Yang Z. Clinical study of a formula for delayed rapid fluid resuscitation for patients with burn shock. Burns. 2005;31(5):617-22.

54. Sudhakar GV, Lakshmi P. Role of HES 130/0.4 in resuscitation of patients with major burn injury. Transfus Altern Transfus Med. 2008;10(2):43-50.
55. Vlachou E, Gosling P, Moiemen NS. Hydroxyethylstarch supplementation in burn resuscitation--a prospective randomised controlled trial. Burns. 2010; 36(7):984-91.

56. Waxman K, Holness R, Tominaga G, Chela P, Grimes J. Hemodynamic and oxygen transport effects of pentastarch in burn resuscitation. Ann Surg. 1989:209(3):341-5.

57. Aoki K, Yoshino A, Yoh K, Sekine K, Yamazaki M, Aikawa N. A comparison of Ringer's lactate and acetate solutions and resuscitative effects on splanchnic dysoxia in patients with extensive burns. Burns. 2010;36(7):1080-5.

58. O'Mara MS, Slater H, Goldfarb IW, Caushaj PF. A prospective, randomized evaluation of intra-abdominal pressures with crystalloid and colloid resuscitation in burn patients. J Trauma. 2005;58(5):1011-8.

59. Tanaka H, Matsuda T, Miyagantani Y, Yukioka T, Matsuda H, Shimazaki S. Reduction of resuscitation fluid volumes in severely burned patients using ascorbic acid administration: a randomized, prospective study. Arch Surg. 2000;135(3):326-31.

60. Bechir M, Puhan MA, Neff SB, Guggenheim M, Wedler V, Stover JF, et al. Early fluid resuscitation with hyperoncotic hydroxyethyl starch 200/0.5 (10\%) in severe burn injury. Crit Care. 2010;14(3):R123.

61. Bocanegra M, Hinostroza F, Kefalides NA, Markley K, Rosenthal SM. A longterm study of early fluid therapy in severely burned adults. 3. Simultaneous comparison of saline solution alone or combined with plasma. JAMA. 1966; 195(4):268-74.

62. Chung KK, Wolf SE, Cancio LC, Alvarado R, Jones JA, McCorcle J, et al. Resuscitation of severely burned military casualties: fluid begets more fluid. J Trauma Inj Infect Crit Care. 2009;67(2):231-7.

63. Jelenko C 3rd, Wheeler ML, Callaway BD, Divilio LT, Bucklen KR, Holdredge TD. Shock and resuscitation. II: Volume repletion with minimal edema using the "HALFD"(Hypertonic Albuminated Fluid Demand) regimen. Jacep. 1978; 7(9):326-33.

64. Murphy JT, Horton JW, Purdue GF, Hunt JL. Cardiovascular effect of 7.5\% sodium chloride-dextran infusion after thermal injury. Arch Surg. 1999; 134(10):1091-7.

65. Oda J, Ueyama M, Yamashita K, Inoue T, Noborio M, Ode Y, et al. Hypertonic lactated saline resuscitation reduces the risk of abdominal compartment syndrome in severely burned patients. J Trauma. 2006;60(1):64-71.

66. Aboelatta Y, Abdelsalam A. Volume overload of fluid resuscitation in acutely burned patients using transpulmonary thermodilution technique. J Burn Care Res. 2013;34(3):349-54.

67. Arlati S, Storti E, Pradella V, Bucci L, Vitolo A, Pulici M. Decreased fluid volume to reduce organ damage: a new approach to burn shock resuscitation? A preliminary study. Resuscitation. 2007;72(3):371-8.

68. Berger MM, Pictet A, Revelly JP, Frascarolo P, Chioléro RL. Impact of a bicarbonated saline solution on early resuscitation after major burns. Intensive Care Med. 2000;26(9):1382-5.

69. Gille J, Klezcewski B, Malcharek M, Raff T, Mogk M, Sablotzki A, et al. Safety of resuscitation with Ringer's acetate solution in severe burn (VolTRAB)--an observational trial. Burns. 2014;40(5):871-80.

70. Salinas J, Chung KK, Mann EA, Cancio LC, Kramer GC, Serio-Melvin ML, et al. Computerized decision support system improves fluid resuscitation following severe burns: an original study. Crit Care Med. 2011;39(9):2031-8.

71. Csontos C, Foldi V, Fischer T, Bogar L. Arterial thermodilution in burn patients suggests a more rapid fluid administration during early resuscitation. Acta Anaesthesiol Scand. 2008;52(6):742-9.

72. Holm C, Mayr M, Tegeler J, Horbrand F. Henckel von Donnersmarck G, Muhlbauer W, et al. A clinical randomized study on the effects of invasive monitoring on burn shock resuscitation. Burns. 2004;30(8):798-807.

73. Tokarik M, Sjoberg F, Balik M, Pafcuga I, Broz L. Fluid therapy LiDCO controlled trial-optimization of volume resuscitation of extensively burned patients through noninvasive continuous real-time hemodynamic monitoring LiDCO. J Burn Care Res. 2013;34(5):537-42.

74. Holm C, Melcer B, Hörbrand F. Henckel von Donnersmarck G, Mühlbauer W. Arterial thermodilution: an alternative to pulmonary artery catheter for cardiac output assessment in burn patients. Burns. 2001;27(2):161-6.

75. Berger MM, Binnert C, Chiolero RL, Taylor W, Raffoul W, Cayeux MC, et al. Trace element supplementation after major burns increases burned skin trace element concentrations and modulates local protein metabolism but not whole-body substrate metabolism. Am J Clin Nutr. 2007:85(5):1301-6.

76. Chen Z, Wang S, Yu B, Li A. A comparison study between early enteral nutrition and parenteral nutrition in severe burn patients. Burns. 2007:33(6): 708-12. 
77. Chuntrasakul C, Siltham S, Sarasombath S, Sittapairochana C, Leowattana W, Chockvivatanavanit $\mathrm{S}$, et al. Comparison of a immunonutrition formula enriched arginine, glutamine and omega-3 fatty acid, with a currently highenriched enteral nutrition for trauma patients. J Med Assoc Thai. 2003;86(6): 552-61.

78. Garcia-de-Lorenzo A, Denia R, Atlan P, Martinez-Ratero S, Le Brun A, Evard $D$, et al. Parenteral nutrition providing a restricted amount of linoleic acid in severely burned patients: a randomised double-blind study of an olive oilbased lipid emulsion v. medium/long-chain triacylglycerols. Br J Nutr. 2005; 94(2):221-30.

79. Garrel DR, Razi M, Lariviere F, Jobin N, Naman N, Emptoz-Bonneton A, et al. Improved clinical status and length of care with low-fat nutrition support in burn patients. JPEN J Parenter Enteral Nutr. 1995;19(6):482-91.

80. Gottschlich MM, Jenkins M, Warden GD, Baumer T, Havens P, Snook JT, et al. Differential effects of three enteral dietary regimens on selected outcome variables in burn patients. JPEN J Parenter Enteral Nutr. 1990; 14(3):225-36.

81. Herndon DN, Barrow RE, Stein M, Linares H, Rutan TC, Rutan R, et al. Increased mortality with intravenous supplemental feeding in severely burned patients. J Burn Care Rehabil. 1989;10(4):309-13.

82. Herndon DN, Stein MD, Rutan TC, Abston S, Linares H. Failure of TPN supplementation to improve liver function, immunity, and mortality in thermally injured patients. J Trauma. 1987;27(2):195-204.

83. Larsson J, Lennmarken C, Martensson J, Sandstedt S, Vinnars E. Nitrogen requirements in severely injured patients. Br J Surg. 1990;77(4):413-6.

84. Ostadrahimi A, Nagili B, Asghari-Jafarabadi M, Beigzali S, Zalouli H, Lak S. A proper enteral nutrition support improves sequential organ failure score and decreases length of stay in hospital in burned patients. Iran Red Crescent Med J. 2016;18(2):e21775.

85. Peng X, Yan H, You Z, Wang P, Wang S. Effects of enteral supplementation with glutamine granules on intestinal mucosal barrier function in severe burned patients. Burns. 2004;30(2):135-9.

86. Saffle JR, Wiebke G, Jennings K, Morris SE, Barton RG. Randomized trial of immune-enhancing enteral nutrition in burn patients. J Trauma. 1997;42(5): 793-800 discussion -2.

87. Tihista S, Echavarria E. Effect of omega 3 polyunsaturated fatty acids derived from fish oil in major burn patients: a prospective randomized controlled pilot trial. Clin Nutr. 2017;37(1):107-12.

88. Vicic VK, Radman M, Kovacic V. Early initiation of enteral nutrition improves outcomes in burn disease. Asia Pac J Clin Nutr. 2013;22(4):543-7.

89. Yan H, Peng X, Huang Y, Zhao M, Li F, Wang P. Effects of early enteral arginine supplementation on resuscitation of severe burn patients. Burns. 2007;33(2):179-84

90. Abribat T, Nedelec B, Jobin N, Garrel DR. Decreased serum insulin-like growth factor-I in burn patients: relationship with serum insulin-like growth factor binding protein-3 proteolysis and the influence of lipid composition in nutritional support. Crit Care Med. 2000;28(7):2366-72.

91. Lam NN, Tien NG, Khoa CM. Early enteral feeding for burned patients--an effective method which should be encouraged in developing countries. Burns. 2008;34(2):192-6.

92. Peck MD, Kessler M, Cairns BA, Chang YH, Ivanova A, Schooler W. Early enteral nutrition does not decrease hypermetabolism associated with burn injury. J Trauma. 2004;57(6):1143-8 discussion 8-9.

93. Peng YZ, Yuan ZQ, Xiao GX. Effects of early enteral feeding on the prevention of enterogenic infection in severely burned patients. Burns. 2001;27(2):145-9.

94. Saffle JR, Larson CM, Sullivan J. A randomized trial of indirect calorimetry-based feedings in thermal injury. J Trauma. 1990;30(7):776-82 discussion 82-3.

95. Wibbenmeyer LA, Mitchell MA, Newel IM, Faucher LD, Amelon MJ, Ruffin TO, et al. Effect of a fish oil and arginine-fortified diet in thermally injured patients. J Burn Care Res. 2006;27(5):694-702.

96. Zhou YP, Jiang ZM, Sun YH, Wang XR, Ma EL, Wilmore D. The effect of supplemental enteral glutamine on plasma levels, gut function, and outcome in severe burns: a randomized, double-blind, controlled clinical trial. JPEN J Parenter Enteral Nutr. 2003;27(4):241-5.

97. Brown $\mathrm{RO}$, Buonpane $\mathrm{EA}$, Vehe $\mathrm{KL}$, Hickerson WL, Luther RW. Comparison of modified amino acids and standard amino acids in parenteral nutrition support of thermally injured patients. Crit Care Med. 1990;18(10):1096-101.

98. Dhanraj P, Chacko A, Mammen M, Bharathi R. Hospital-made diet versus commercial supplement in postburn nutritional support. Burns. 1997;23(6): $512-4$.
99. Falder S, Silla R, Phillips M, Rea S, Gurfinkel R, Baur E, et al. Thiamine supplementation increases serum thiamine and reduces pyruvate and lactate levels in burn patients. Burns. 2010;36(2):261-9.

100. Hiebert JM, Brown A, Anderson RG, Halfacre S, Rodeheaver GT, Edlich RF. Comparison of continuous vs intermittent tube feedings in adult burn patients. Jpen J Parenter Enter Nutr. 5(1):73-5.

101. Shields BA, Brown JN, Aden JK, Salgueiro M, Mann-Salinas EA, Chung KK. A pilot review of gradual versus goal re-initiation of enteral nutrition after burn surgery in the hemodynamically stable patient. Burns. 2014;40(8):1587-92.

102. Gudaviciene D, Rimdeika R, Adamonis K. Influence of enteral nutrition on the frequency of complications in case of major burns. Medicina (Kaunas, Lithuania). 2004:40(10):957-61.

103. Kesey J, Dissanaike S. A protocol of early aggressive acceleration of tube feeding increases ileus without perceptible benefit in severely burned patients. J Burn Care Res. 2013;34(5):515-20.

104. Soguel L, Chiolero RL, Ruffieux C, Berger MM. Monitoring the clinical introduction of a glutamine and antioxidant solution in critically ill trauma and burn patients. Nutrition. 2008;24(11-12):1123-32

105. Varon DE, Freitas G, Goel N, Wall J, Bharadia D, Sisk E, et al. Intraoperative feeding improves calorie and protein delivery in acute burn patients. J Burn Care Res. 2017;38(5):299-303.

106. Rutan TC, Herndon DN, Van Osten T, Abston S. Metabolic rate alterations in early excision and grafting versus conservative treatment. J Trauma. 1986; 26(2):140-2.

107. Sorensen B. Acute excision or exposure treatment? Results of a randomized controlled clinical trial. Scand J Plast Reconstr Surg. 1979;13(1):115-8.

108. Guo ZR, Sheng CY, Diao L, Gao WY, Yang HM, Lin HY, et al. Extensive wound excision in the acute shock stage in patients with major burns. Burns. 1995:21(2):139-42.

109. Kisslaogglu E, Yuksel F, Uccar C, Karacaogglu E. Rationale for early tangential excision and grafting in burn patients. Acta Chirurgiae Plasticae. 1997;39(1):9-12.

110. Puri V, Khare NA, Chandramouli M, Shende N, Bharadwaj S. Comparative analysis of early excision and grafting vs delayed grafting in burn patients in a developing country. J Burn Care Res. 2016;37(5):278-82.

111. Johansson PI, Eriksen K, Nielsen SL, Rojkjaer R, Alsbjorn B. Recombinant FVlla decreases perioperative blood transfusion requirement in burn patients undergoing excision and skin grafting--results of a single centre pilot study. Burns. 2007;33(4):435-40

112. Mzezewa S, Jonsson K, Aberg M, Sjoberg T, Salemark L. A prospective double blind randomized study comparing the need for blood transfusion with terlipressin or a placebo during early excision and grafting of burns. Burns. 2004;30(3):236-40.

113. Palmieri TL, Holmes JH, Arnoldo B, Peck M, Potenza B, Cochran A, et al. Transfusion requirement in burn care evaluation (TRIBE): a multicenter randomized prospective trial of blood transfusion in major burn injury. Ann Surg. 2017:266(4):595-602

114. Schaden E, Kimberger O, Kraincuk P, Baron DM, Metnitz PG, KozekLangenecker $\mathrm{S}$. Perioperative treatment algorithm for bleeding burn patients reduces allogeneic blood product requirements. Br J Anaesth. 2012 109(3):376-81.

115. Still JM Jr, Belcher K, Law EJ, Thompson W, Jordan M, Lewis M, et al. A double-blinded prospective evaluation of recombinant human erythropoietin in acutely burned patients. J Trauma. 1995;38(2):233-6.

116. Lundy JB, Hetz K, Chung KK, Renz EM, White CE, King BT, et al. Outcomes with the use of recombinant human erythropoietin in critically ill burn patients. Am Surg. 2010;76(9):951-6.

117. Imai R, Matsumura H, Uchida R, Watanabe K. Perioperative hemodilutional autologous blood transfusion in burn surgery. Injury. 39(1):57-60.

118. Kowal-Vern A, McGill V, Walenga JM, Gamelli RL. Antithrombin( $\mathrm{H})$ concentrate infusions are safe and effective in patients with thermal injuries. J Burn Care Rehabil. 2000;21(2):115-27.

119. Elsharnouby NM, Eid HE, Abou Elezz NF, Aboelatta YA. Heparin/Nacetylcysteine: an adjuvant in the management of burn inhalation injury: a study of different doses. J Crit Care. 2014;29(1):182-e1-4.

120. Reper $P$, Wibaux $O$, Van Laeke $P$, Vandeenen D, Duinslaeger L, Vanderkelen A. High frequency percussive ventilation and conventional ventilation after smoke inhalation: a randomised study. Burns. 2002;28(5):503-8.

121. Chung KK, Wolf SE, Renz EM, Allan PF, Aden JK, Merrill GA, et al. Highfrequency percussive ventilation and low tidal volume ventilation in burns: a randomized controlled trial. Crit Care Med. 2010;38(10):1970-7. 
122. McGinn KA, Weigartz K, Lintner A, Scalese MJ, Kahn SA. Nebulized heparin with $\mathrm{N}$-acetylcysteine and albuterol reduces duration of mechanical ventilation in patients with inhalation injury. J Pharm Pract. 2019:32(2):163-6.

123. Miller AC, Rivero A, Ziad S, Smith DJ, Elamin EM. Influence of nebulized unfractionated heparin and $\mathrm{N}$-acetylcysteine in acute lung injury after smoke inhalation injury. J Burn Care Res. 2009;30(2):249-56.

\section{Publisher's Note}

Springer Nature remains neutral with regard to jurisdictional claims in published maps and institutional affiliations.

Ready to submit your research? Choose BMC and benefit from:

- fast, convenient online submission

- thorough peer review by experienced researchers in your field

- rapid publication on acceptance

- support for research data, including large and complex data types

- gold Open Access which fosters wider collaboration and increased citations

- maximum visibility for your research: over $100 \mathrm{M}$ website views per year

At $\mathrm{BMC}$, research is always in progress.

Learn more biomedcentral.com/submissions 